\title{
Spaces of nonnegatively curved surfaces
}

\author{
By Taras BANAKH and Igor BelegradeK
}

(Received Oct. 25, 2015)

(Revised Oct. 22, 2016)

\begin{abstract}
We determine the homeomorphism type of the space of smooth complete nonnegatively curved metrics on $S^{2}, R P^{2}$, and $\mathbb{C}$ equipped with the topology of $C^{\gamma}$ uniform convergence on compact sets, when $\gamma$ is infinite or is not an integer. If $\gamma=\infty$, the space of metrics is homeomorphic to the separable Hilbert space. If $\gamma$ is finite and not an integer, the space of metrics is homeomorphic to the countable power of the linear span of the Hilbert cube. We also prove similar results for some other spaces of metrics including the space of complete smooth Riemannian metrics on an arbitrary manifold.
\end{abstract}

\section{Introduction.}

In this paper smooth means $C^{\infty}$, by a manifold we mean a smooth finite-dimensional manifold without boundary, the $C^{\gamma}$ topology on a set of maps between two fixed manifolds is the topology of uniform $C^{\gamma}$ convergence on compact smooth domains, where $0 \leq \gamma \leq$ $\infty$, see Section 2. The $C^{\gamma}$ topology is called Hölder if $\gamma$ is finite and not an integer. Note that the $C^{\gamma}$ topology on any set of smooth maps is metrizable and separable.

Given a connected manifold $M$ of positive dimension let $\mathcal{R}^{\gamma}(M)$ denote the space of all complete smooth Riemannian metrics on $M$ endowed with the $C^{\gamma}$ topology. For finite $\gamma$ the mismatch between infinite smoothness of the metrics and their $C^{\gamma}$ convergence results in a larger number of converging sequences. This situation arises naturally in applications, e.g., according to [AC92] if a sequence of smooth metrics $g_{i}$ on a closed manifold has uniform lower bounds on injectivity radius and Ricci curvature as well as an upper diameter bound, then for some diffeomorphisms $\phi_{i}$ the family of pullback metrics $\phi_{i}^{*} g_{i}$ is $C^{\gamma}$ precompact for every $\gamma<1$, see [Pet97] for other results of this type.

Our first result recognizes the homeomorphism type of $\mathcal{R}^{\gamma}(M)$ :

THEOREM 1.1. If $M$ is a connected manifold of positive dimension, then $\mathcal{R}^{\infty}(M)$ is homeomorphic to $\ell^{2}$, and $\mathcal{R}^{\gamma}(M)$ is homeomorphic to $\Sigma^{\omega}$ for every finite $\gamma$.

Here $\ell^{2}$ is the separable Hilbert space, $\Sigma$ is the linear span of the standard Hilbert cube in $\ell^{2}$, and $\Sigma^{\omega}$ is the product of countably many copies of $\Sigma$. Since the space $\Sigma^{\omega}$ may be unfamiliar to the reader, let us mention that it is a locally convex linear space which is a countable union of nowhere dense sets, so $\Sigma^{\omega}$ is not completely metrizable. If $\Omega$ is either $\ell^{2}$ or $\Sigma^{\omega}$, then it has the following properties, see Section 3:

2010 Mathematics Subject Classification. Primary 53C21; Secondary 57N20.

Key Words and Phrases. nonnegative curvature, space of metrics, infinite dimensional topology, absorbing. 
(a) $\Omega$ is not $\sigma$-compact, and in particular, not locally compact.

(b) Any two open homotopy equivalent subsets of $\Omega$ are homeomorphic.

(c) The complement to any compact subset of $\Omega$ is contractible.

(d) Any homeomorphism of two compact subsets of $\Omega$ extends to a homeomorphism of $\Omega$.

In (c) and (d) one can replace the phrase "compact subset" by " $Z$-set"; many examples of $Z$-sets can be found in Section 3 .

A starting point in the proof of Theorem 1.1 is the convexity of $\mathcal{R}^{\gamma}(M)$ in the locally convex linear space of all smooth symmetric 2-tensors on $M$, with the $C^{\gamma}$ topology. As a caution we note that there is no meaningful homeomorphism classification of convex subsets of locally convex linear spaces, e.g., the union of an open ball in $\ell^{2}$ with any subset of its boundary sphere is convex. Such pathologies do not occur in the case at hand. There is a standard machinery that makes recognizing the homeomorphism type of $\mathcal{R}^{\infty}(M)$ quite easy. The case of finite $\gamma$ is more delicate and it exploits a certain argument in [Ban00] together with a substantial amount of established techniques.

For a constant $\lambda$ let $\mathcal{R}_{\geq \lambda}^{\gamma}(M)$ denote the subspace of $\mathcal{R}^{\gamma}(M)$ consisting of the metrics with sectional curvature $\geq \lambda$; the space $\mathcal{R}_{>\lambda}^{\gamma}(M)$ is defined similarly. The subspaces are generally non-convex in $\mathcal{R}^{\gamma}(M)$, and little is known about their topological properties. A natural idea is to find a homeomorphic parametrization of $\mathcal{R}_{\geq \lambda}^{\gamma}(M)$ by a space to which techniques of infinite dimensional topology apply. In this paper we do that when $M$ is $\mathbb{C}$, $S^{2}$ or $R P^{2}$, the surfaces admitting a complete non-flat metric of nonnegative curvature, provided $\gamma \notin \mathbb{Z}$ and also $\lambda=0$ for $M=\mathbb{C}$. Here are our main results:

TheOREM 1.2. If $M$ is $S^{2}$ or $R P^{2}$, then for every real $\lambda$ the spaces $\mathcal{R}_{\geq_{\lambda}}^{\gamma}(M)$ and $\mathcal{R}_{>\lambda}^{\gamma}(M)$ are homeomorphic to

(1) $\ell^{2}$ if $\gamma=\infty$,

(2) $\Sigma^{\omega}$ if $\gamma$ is finite and not an integer.

TheOrem 1.3. The spaces $\mathcal{R}_{\geq 0}^{\gamma}(\mathbb{C})$ and $\mathcal{R}_{>0}^{\gamma}(\mathbb{C})$ are homeomorphic to

(1) $\ell^{2}$ if $\gamma=\infty$,

(2) $\Sigma^{\omega}$ if $\gamma$ is finite and not an integer.

The homeomorphism of $\mathcal{R}_{\geq 0}^{\infty}(\mathbb{C})$ and $\ell^{2}$ was already established in [BH15].

To explain the assumption $\gamma \notin \mathbb{Z}$, let us sketch the analytic ingredients of Theorem 1.3 in the case of $\mathcal{R}_{\geq 0}^{\gamma}(\mathbb{C})$. It is well-known, see [BF42], that any complete metric of nonnegative curvature on $\mathbb{C}$ is conformally equivalent to the standard Euclidean metric $g_{0}$. Hence it can be written as $\phi^{*} e^{-2 u} g_{0}$ where $\phi$ is an orientation-preserving diffeomorphism of $\mathbb{C}$ and $u$ is a smooth function on $\mathbb{C}$. By composing $\phi$ with a conformal automorphism of $\mathbb{C}$ one can assume that $\phi$ fixes a pair of points, say 0 and 1 , which determines $\phi$ uniquely. Such $\phi$ 's form a closed contractible subgroup of the diffeomorphism group of $\mathbb{C}$, which we denote $D^{\gamma+1}(\mathbb{C})$; the superscript $\gamma+1$ indicates that the 
group is equipped with the $C^{\gamma+1}$ topology, which is natural because $\phi^{*} e^{-2 u} g_{0}$ involves the differential of $\phi$. Nonnegativity of the curvature is equivalent to subharmonicity of $u$. Completeness of $e^{-2 u} g_{0}$ imposes further restrictions on $u$, making it roughly speaking of sublogorithmic growth, and such $u$ 's form a convex subset $\mathcal{O}_{\geq 0}^{\gamma}(\mathbb{C})$ in the space of smooth functions equipped with the $C^{\gamma}$ topology, see [BH15]. With the above restrictions on $\phi$ and $u$ the map $(\phi, u) \rightarrow \phi^{*} e^{-2 u} g_{0}$ becomes a continuous bijection. Moreover, smooth dependence of solutions of Beltrami equation on the dilatation shows that the continuous bijection is a homeomorphism for $\gamma \notin \mathbb{Z}$, see [BH15], [BH16]; we expect this to fail when $\gamma$ is an integer. In summary, for $\gamma \notin \mathbb{Z}$ the space $\mathcal{R}_{>0}^{\gamma}(\mathbb{C})$ is homeomorphic to the product $D^{\gamma+1}(\mathbb{C}) \times \mathcal{O}_{>0}^{\gamma}(\mathbb{C})$ where $D^{\gamma+1}(\mathbb{C})$ is a certain diffeomorphism group and $\mathcal{O}_{\geq 0}^{\gamma}(\mathbb{C})$ is some convex set of smooth functions on $\mathbb{C}$. The homeomorphism type of the factors $D^{\gamma+1}(\mathbb{C}), \mathcal{O}_{\geq 0}^{\gamma}(\mathbb{C})$ can be determined along the lines of the proof of Theorem 1.1, and the conclusion is that $D^{\gamma+1}(\mathbb{C}), \mathcal{O}_{\geq 0}^{\gamma}(\mathbb{C})$ are each homeomorphic to $\ell^{2}$ or $\Sigma^{\omega}$ depending on whether $\gamma$ is infinite or finite. The case $\mathcal{R}_{>0}^{\gamma}(\mathbb{C})$ is similar, and the proof of Theorem 1.2 follows the same outline except that [BH15] is not needed.

The above proof requires that all metrics lie in the same conformal class, and in particular, the proof does not extend to $\mathcal{R}_{\geq \lambda}^{\gamma}(\mathbb{C})$ with $\lambda<0$ or to $\mathcal{R}_{\geq \lambda}^{\gamma}(M)$ where $M$ is a closed surface of nonpositive Euler characteristic. The space $\mathcal{R}_{\geq \lambda}^{\gamma}(\mathbb{C})$ with $\lambda>0$ is empty due to the Myers theorem: Any complete Riemannian manifold of curvature $\geq \lambda>0$ is compact. Another essential feature of the proof is the convexity of $\mathcal{O}_{\geq 0}^{\gamma}(\mathbb{C})$ which prevents us from treating spaces of metrics with two sided curvature bounds such as the subspace of $\mathcal{R}_{\geq 0}^{\gamma}(\mathbb{C})$ consisting of metrics with curvature $\leq 1$.

Techniques of this paper apply to other convex sets of smooth maps not necessarily equipped with the $C^{\gamma}$ topology, and whenever possible the results are stated so that they can be easily used in other contexts, see Sections $4-5$.

Structure of the paper. Section 2 is a review of definitions and properties of the $C^{\gamma}$ topology. The necessary infinite dimensional topology background is collected in Section 3. Sections 4 and 5 contain the main technical results needed to treat the case when $\gamma$ is finite. Theorem 1.1 is proved in Sections 6 and 7. Basic information on the spaces of metrics on surfaces is collected in Sections 8 and 9. Finally, Theorems 1.2-1.3 are proved in Sections 10-12.

Acknowledgments. We are thankful to the referee for editorial suggestions. Belegradek is grateful for NSF support: DMS-1105045. Some of the ideas in the present paper go back to Belegradek's joint work with Jing $\mathrm{Hu}$, which appeared in her thesis $[\mathbf{H u 1 5}$. The results we prove here are much stronger, e.g., one of the main theorems in [Hu15] is the topological homogeneity of $\mathcal{R}_{>0}^{\gamma}\left(S^{2}\right)$ when $\gamma \geq 2$ and $\gamma \notin \mathbb{Z}$, while we determine the homeomorphism type of $\mathcal{R}_{\geq 0}^{\gamma}\left(S^{2}\right)$ for $\gamma \notin \mathbb{Z}$.

\section{A review of $C^{\gamma}$ topology.}

In this section we recall definitions and basic properties of the $C^{\gamma}$ topology. We stress that smooth always means $C^{\infty}$. Fix $\gamma \in[0, \infty]$, and if $\gamma$ is finite write it uniquely as $\gamma=k+\alpha$ where $k$ is a nonnegative integer and $\alpha \in[0,1)$. 
If $\alpha=0$, then the linear space $C^{k}\left(D, \mathbb{R}^{n}\right)$ of $k$-times continuously differentiable maps from a smooth compact domain $D \subset \mathbb{R}^{m}$ to $\mathbb{R}^{n}$ is Banach with respect to the norm

$$
\|f\|_{C^{k}\left(D, \mathbb{R}^{n}\right)}=\max \left\{\left|f^{(p)}(x)\right|:|p| \leq k, x \in D\right\}
$$

where $f^{(p)}$ is a partial derivative for the multi-index $p$ of order $|p|$. If $\alpha \in(0,1)$, we say that a map $f \in C^{0}\left(D, \mathbb{R}^{n}\right)$ is $\alpha$-Hölder if the following quantity is finite

$$
\|f\|_{C^{\alpha}\left(D, \mathbb{R}^{n}\right)}=\sup \left\{\frac{|f(x)-f(y)|}{|x-y|^{\alpha}}: x, y \in D, x \neq y\right\}
$$

where $|\cdot|$ is the Euclidean norm. The maps in $C^{k}\left(D, \mathbb{R}^{n}\right)$ whose $k$-th partial derivatives are all $\alpha$-Hölder form a linear subspace, denoted $C^{k+\alpha}\left(D ; \mathbb{R}^{n}\right)$, which is Banach with respect to the norm

$$
\|f\|_{C^{k+\alpha}\left(D, \mathbb{R}^{n}\right)}=\|f\|_{C^{k}\left(D, \mathbb{R}^{n}\right)}+\sup _{|\mu|=k}\left\|D^{\mu} f\right\|_{C^{\alpha}\left(D, \mathbb{R}^{n}\right)} .
$$

A map of smooth manifolds is called $C^{k+\alpha}$ if whenever it is written in local coordinates as a map between open sets of Euclidean spaces its restriction to any compact smooth domain has the property that all $k$-th partial derivatives are $\alpha$-Hölder. With this definition the elements of $C^{k+\alpha}\left(D, \mathbb{R}^{n}\right)$ are precisely the $C^{k+\alpha}$ maps from $D$ to $\mathbb{R}^{n}$ because if $D^{\prime}$ is another compact domain in $\mathbb{R}^{m}$, then the composite of a map in $C^{\infty}\left(D^{\prime}, D\right)$ with a map in $C^{k+\alpha}\left(D, \mathbb{R}^{n}\right)$ is in $C^{k+\alpha}\left(D^{\prime}, \mathbb{R}^{n}\right)$, see [BHS05, Section 2.2].

Clearly any $C^{k+\alpha}$ map is $C^{k}$. Less trivially, for every $0<\gamma<\beta \leq \infty$ any $C^{\beta}$ map is $C^{\gamma}$, see [GT01, Section 6.8] for the case when $\gamma, \beta$ are not integers. The product of two real valued $C^{k+\alpha}$ functions is $C^{k+\alpha}$, see [GT01, Section 4.1]. If $k \geq 1$, then the composition of $C^{k+\alpha}$ maps is $C^{k+\alpha}$, and the inverse of an invertible $C^{k+\alpha}$ map is $C^{k+\alpha}$, see [BHS05, Sections 2.1-2.2].

We are now ready to define the $C^{\gamma}$ topology on the set $C^{\gamma}(M, N)$ of $C^{\gamma}$ maps between smooth manifolds $M, N$, cf. [Pal68, Chapters 7-8]. Fix a smooth embedding $i_{N}$ of $N$ as a closed submanifold of some $\mathbb{R}^{n}$. Postcomposing with $i_{N}$ defines an inclusion of $C^{\gamma}(M, N)$ into $C^{\gamma}\left(M, \mathbb{R}^{n}\right)$. We shall define the $C^{\gamma}$ topology on $C^{\gamma}\left(M, \mathbb{R}^{n}\right)$ and then give $C^{\gamma}(M, N)$ the subspace topology. Cover $M$ by a countable family of smooth compact domains $D_{j}$ each lying in some coordinate chart. The Banach norm $\|\cdot\|_{C^{k+\alpha}\left(D_{j}, \mathbb{R}^{n}\right)}$ on $C^{\gamma}\left(D_{j}, \mathbb{R}^{n}\right)$ defines a seminorm $p_{j}$ on $C^{\gamma}\left(M, \mathbb{R}^{n}\right)$ by restriction, i.e., $p_{j}(f)=\left\|\left.f\right|_{D_{j}}\right\|_{C^{k+\alpha}\left(D_{j}, \mathbb{R}^{n}\right)}$. The countable family of seminorms $p_{j}$ gives $C^{\gamma}\left(M, \mathbb{R}^{n}\right)$ a Fréchet space structure, and one can show that the structure is independent of the choices involved.

The space $C^{\infty}\left(M, \mathbb{R}^{n}\right)=\bigcap_{k \in \mathbb{N}} C^{k}\left(M, \mathbb{R}^{n}\right)$ gets the associated Fréchet structure given by seminorms $\|\cdot\|_{C^{k}\left(D_{j}, \mathbb{R}^{n}\right)}$ where both $k$ and $j$ vary. More details can be found in [Trè67, Chapter 10, Example 1] and [Hir94, Sections 1 and 4 in Chapter 2].

Both $C^{k}\left(M, \mathbb{R}^{n}\right)$ and $C^{\infty}\left(M, \mathbb{R}^{n}\right)$ are separable, see e.g. [BH15, Lemma 3.2], while it is well-known that $C^{k+\alpha}\left(M, \mathbb{R}^{n}\right)$ is non-separable if $\alpha \neq 0$, except when $\operatorname{dim} M$ or $n$ vanish. 


\section{Infinite dimensional topology background.}

In this section any space is assumed metrizable and separable, and any map is assumed continuous; we do not follow the convention in other sections.

By a subspace we always mean "subset with subspace topology"; we never use the term to mean a linear subspace.

A basic goal of infinite-dimensional topology is to give a homeomorphism classification of naturally occurring spaces such as topological groups, linear spaces and their convex subsets. A closely related problem is a characterization of $\Omega$-manifolds for a suitable space $\Omega$. Here an $\Omega$-manifold is a space in which every point has a neighborhood homeomorphic to an open subset of $\Omega$. Satisfactory answers are known, e.g., when $\Omega$ is $\ell^{2}$ or $\Omega$ is absorbing in the sense of Bestvina-Mogilski [BM86].

The exposition below follows [BP75], [BRZ96], [BM86], [DM90]. The subject is quite technical so we attempt to limit the terminology to what is relevant for our applications.

A linear metric space is a vector space with a translation-invariant metric. A linear metric space is locally convex if its topology is given by a countable family of seminorms. Any normed space is locally convex. A Fréchet space is a complete locally convex linear metric space. The dimension of a convex subset $C$ is the dimension of the vector space affinely isomorphic to the affine hull $\operatorname{Aff} C$ of $C$.

Any convex set in a locally convex linear metric space is an AR (absolute retract), see [BP75, Corollary II.5.3]. A space is an AR if and only if it is a contractible ANR (absolute neighborhood retract), see [BP75, Theorem II.5.1]. A space that is locally an ANR is an ANR [BP75, Theorem II.5.1].

A subset $B \subset X$ is homotopy dense if there is a homotopy $h: X \times I \rightarrow X$ with $h_{0}=$ id and $h(X \times(0,1]) \subset B$. An embedding with a homotopy dense image is homotopy dense. A subset is homotopy negligible if its complement is homotopy dense. If $X$ is an ANR, then a subset $B$ of $X$ is homotopy dense if and only if each map $I^{n} \rightarrow X$ with $\partial I^{n} \subset B$ can be uniformly approximated rel boundary by maps $I^{n} \rightarrow B$, see [BRZ96, Theorem 1.2.2]. Other characterizations of homotopy dense subsets can be found in [BRZ96, Exercise 12 in 1.2].

It follows that a subset $B$ is homotopy dense if and only if it is locally homotopy dense, see [BRZ96, Exercise 3 in 1.2]; the latter means that each point has a neighborhood $U$ such that $U \cap B$ is homotopy dense in $U$.

Any homotopy dense subset of an $\ell^{2}$-manifold is infinite-dimensional [BRZ96, Exercise 6 in 1.3]. Any homotopy dense subset of an ANR is an ANR, so in particular, any homotopy dense subset of an $\ell^{2}$-manifold is an ANR.

Given an open cover $\mathcal{U}$ two maps $f, g: Y \rightarrow X$ are $\mathcal{U}$-close if for every $y \in Y$ there is $U \in \mathcal{U}$ with $f(y), g(y) \in U$. Let $Q$ denote the Hilbert cube.

A space $X$ has the Strong Discrete Approximation Property (SDAP) if for every open cover $\mathcal{U}$ of $X$ each map $Q \times \mathbb{N} \rightarrow X$ is $\mathcal{U}$-close to a map $g: Q \times \mathbb{N} \rightarrow X$ such that every point of $X$ has a neighborhood that intersects at most one set of the family $\{g(Q \times\{n\})\}_{n \in \mathbb{N}}$. A space is homeomorphic to a homotopy dense subset of an $\ell^{2}$-manifold if and only if it is an ANR with SDAP [BRZ96, Theorem 1.3.2].

The following is proved in [BRZ96, Propositions 5.2.1 and 5.2.6]: 
Lemma 3.1. Let $C$ be a convex subset of a separable Fréchet space. If the closure $\bar{C}$ of $C$ is either not locally compact, or not contained in $\mathrm{Aff} C$, then $C$ is homeomorphic to a homotopy dense subset of $\ell^{2}$.

A closed subset $B$ of a space $X$ is a $Z$-set if every map $Q \rightarrow X$ can be uniformly approximated by a map whose range misses $B$. A space is called $\sigma Z$ if it is a countable union of $Z$-sets. If $X$ is an ANR, then a subset $B$ of $X$ is a $Z$-set if and only if $B$ is closed and homotopy negligible, see [BRZ96, Theorem 1.4.4] and [BP75, Proposition V.2.1].

ExAMPLE 3.2. (1) The union of a locally finite family of $Z$-sets in an ANR is a $Z$-set [BRZ96, Exercise 1 in 1.4].

(2) If $X, Y$ are ANR and $B$ is a $Z$-set in $X$, then $B \times Y$ is a $Z$-set in $X \times Y$.

(3) If $X$ is homeomorphic to a homotopy dense subset of an $\ell^{2}$-manifold, then any compact subset is a $Z$-set [BRZ96, Proposition 1.4.9].

There are some variations of the notion of a $Z$-set that are all equivalent when the ambient space is a homotopy dense subset of an $\ell^{2}$-manifold, see [BRZ96, Section 1.4] and [BP75, Proposition V.2.1]. In particular, a strong $Z$-set in a space $X$ is a closed subset $A$ of $X$ such that for any open cover $\mathcal{U}$ there is a map $f: X \rightarrow X$ that is $\mathcal{U}$-close to the identity and such that $A$ is disjoint from the closure of $f(X)$. Actually, we shall never use the definition because any strong $Z$-set is a $Z$-set, while in a homotopy dense subset of an $\ell^{2}$-manifold any $Z$-set is strong, see [BRZ96, Proposition 1.4.3]. If an ANR is a countable union of strong $Z$-sets, then it admits a homotopy dense embedding into an $\ell^{2}$-manifold, see [BM86, Proposition 1.9] and [BRZ96, Proposition 1.4.10].

Every $Z$-set is nowhere dense (otherwise, its closure, which equals to the $Z$-set itself, contains an open set $U$ and the constant map from the Hilbert cube to $U$ cannot be approximated by maps whose range misses $U$ ).

A map is a $Z$-embedding if its image is a $Z$-set. If $\mathcal{C}$ is a class of spaces, then a space $X$ is strongly $\mathcal{C}$-universal if for every open cover $\mathcal{U}$ of $X$, every $C \in \mathcal{C}$, every closed subset $B \subset C$, and every map $f: C \rightarrow X$ that restricts to a $Z$-embedding on $B$ there is a $Z$-embedding $\bar{f}: C \rightarrow X$ with $\left.\bar{f}\right|_{B}=\left.f\right|_{B}$ such that $f, \bar{f}$ are $\mathcal{U}$-close. Note that if $X$ is strongly $\mathcal{C}$-universal, then any space in $\mathcal{C}$ is homeomorphic to a $Z$-set of $X$.

Given a class $\mathcal{C}$ of spaces, a space $X$ is $\mathcal{C}$-absorbing if the following holds:

(i) $X$ is strongly $\mathcal{C}$-universal,

(ii) $X$ is the union of countably many $Z$-sets,

(iii) $X$ is homeomorphic to a homotopy dense subset of an $\ell^{2}$-manifold,

(iv) $X$ is the union of a countably many closed subsets homeomorphic to spaces in $\mathcal{C}$.

ExAmple 3.3. Since $Z$-sets are nowhere dense, any $\mathcal{C}$-absorbing space is meager in itself. Therefore by the Baire category theorem no $\mathcal{C}$-absorbing space is locally compact or completely metrizable (no matter what $\mathcal{C}$ is). In particular, $\ell^{2}$ is not $\mathcal{C}$-absorbing. 
ExAmple 3.4. The linear span $\Sigma$ of the Hilbert cube in $\ell^{2}$ is $\mathcal{M}_{0}$-absorbing, where $\mathcal{M}_{0}$ is the class of compact spaces, see $[\mathbf{M o g} \mathbf{8 4}$, Theorem 6]. Note that $\Sigma$ is $\sigma$-compact.

ExAmple 3.5. The space $\Sigma^{\omega}$ is $\mathcal{M}_{2}$-absorbing, where $\mathcal{M}_{2}$ be the class of spaces homeomorphic to $F_{\sigma \delta}$-sets in compacta, see [BRZ96, Exercise 3 in 2.4]. Note that $\Sigma^{\omega}$ is not $\sigma$-compact (the product of infinitely many $\sigma$-compact noncompact spaces is never $\sigma$-compact).

The term "absorbing" seem to have come from the following property: If $\mathcal{C}$ is the class of compact spaces, and $X$ is a $\mathcal{C}$-absorbing space embedded into $\ell^{2}$, then any compact subset of $\ell^{2}$ can be mapped into $X$ by a homeomorphism of $\ell^{2}$, see [BP75, Theorem V.6.2].

Absorbing spaces have remarkable properties some of which are listed below (in a weakened form). Suppose $\mathcal{C}$ is a class of spaces that is closed under homeomorphisms, passing to closed subsets, and closed-unions (the last property means that if $C$ is the union of two closed subsets that belong to $\mathcal{C}$, then $C \in \mathcal{C}$ ). Suppose also that $\mathcal{C}$ is local, i.e., a space $X$ belongs to $\mathcal{C}$ if and only if each point of $X$ has a neighborhood that belongs to $\mathcal{C}$. Then the following holds:

- (Uniqueness) Any two homotopy equivalent $\mathcal{C}$-absorbing spaces are homeomorphic, see [BM86, Theorem 3.1].

- ( $Z$-set unknotting) If $A, B$ are $Z$-sets in a $\mathcal{C}$-absorbing space $X$, then any homeomorphism $A \rightarrow B$ that is homotopic to the inclusion of $A$ into $X$ extends to a homeomorphism of $X$ [BM86, Theorem 3.2].

- If $X$ is $\mathcal{C}$-absorbing, then $X$ is $\mathcal{F}_{0}(X)$-absorbing, where $\mathcal{F}_{0}(X)$ is the class of spaces homeomorphic to closed subsets of $X$ [BC00, Proposition 1.11]. In particular, every closed subset of $X$ admits a $Z$-embedding into $X$.

- If $\Omega$ is an $\mathcal{C}$-absorbing $\mathrm{AR}$, then one has:

(1) At each point of a $\mathcal{C}$-absorbing space $X$ there is a local basis of opens sets homeomorphic to $\Omega$.

(2) A space $X$ is $\mathcal{C}$-absorbing if and only if $X$ is an $\Omega$-manifold.

(3) Any $\Omega$-manifold is homeomorphic to an open subset of any other $\Omega$-manifold.

The assertions (2)-(3) are from [BRZ96, Exercise 4 in 1.6], and for completeness we now prove (1). If $X$ is $\mathcal{C}$-absorbing, then $X$ can be identified with a homotopy dense subset of an $\ell^{2}$-manifold $L$, so for any open $U \subset L$ the set $U \cap X$ is homotopy dense in $U$ [BRZ96, Corollary 1.2.3]. Hence if $U$ is contractible, then so is $U \cap X$ (because the inclusion of a homotopy dense subset is a homotopy equivalence). Since $L$ has a local basis of contractible open sets, so does $X$. Each of the contractible open sets is homeomorphic to $\Omega$ by uniqueness of homotopy equivalent $\mathcal{C}$-absorbing spaces. 


\section{4. $\mathcal{M}_{2}$-universality of some functions spaces.}

The main result of this section is Corollary 4.9 establishing $\mathcal{M}_{2}$-universality of a certain class of function spaces. Throughout this section $n$ is a nonnegative integer. Fix a manifold $V$ exhausted by an increasing sequence of compact domains $D_{j}$. As in Section 2 we equip the linear space $C^{n}(V)$ of $n$-times continuously differentiable real valued functions on $V$ with the Fréchet space structure given by the family of seminorms

$$
\|f\|_{C^{n}\left(D_{j}\right)}=\max \left\{\left|f^{(k)}(x)\right|: k \leq n, x \in D_{j}\right\} .
$$

This family of seminorms also endows $C^{\infty}(V)=\bigcap_{n} C^{n}(V)$ with a Fréchet space structure.

Let $\mathcal{M}_{0}$ be the class of metrizable compacta, let $\mathcal{M}_{1}$ be the class of Polish (i.e., completely metrizable) spaces, and let $\mathcal{M}_{2}$ be the class of topological spaces that are homeomorphic to $F_{\sigma \delta}$-sets in metrizable compacta. Note that $\mathcal{M}_{0} \subset \mathcal{M}_{1} \subset \mathcal{M}_{2}$. By $\left(\mathcal{M}_{0}, \mathcal{M}_{2}\right)$ we denote the class of pairs $(K, M)$ where $K$ is a compact metrizable space and $M$ is an $F_{\sigma \delta}$-set in $K$.

A space $X$ is called $\mathcal{M}_{2}$-universal if each space in $\mathcal{M}_{2}$ is homeomorphic to a closed subset of $X$. A pair $(Y, X)$ of spaces with $X \subset Y$ is called

- $\left(\mathcal{M}_{0}, \mathcal{M}_{2}\right)$-universal if for any pair $(K, M) \in\left(\mathcal{M}_{0}, \mathcal{M}_{2}\right)$ there exists a topological embedding $f: K \rightarrow Y$ such that $f^{-1}(X)=M$;

- $\left(\mathcal{M}_{0}, \mathcal{M}_{2}\right)$-preuniversal if for any pair $(K, M) \in\left(\mathcal{M}_{0}, \mathcal{M}_{2}\right)$ there exists a continuous map $f: K \rightarrow Y$ such that $f^{-1}(X)=M$.

The following fundamental result is proved in [BRZ96, Theorems 3.1.1 and 3.2.11]: $A$ subspace $X$ of a Polish space $Y$ is $\mathcal{M}_{2}$-universal if and only if $(Y, X)$ is $\left(\mathcal{M}_{0}, \mathcal{M}_{2}\right)$ universal if and only if $(Y, X)$ is $\left(\mathcal{M}_{0}, \mathcal{M}_{2}\right)$-preuniversal.

The following standard fact can be found in [BRZ96, Exercises 12-13 in 1.2].

Lemma 4.2. Let $L$ be a locally convex space, $X$ be a metrizable convex set in $L$ and $D$ is a dense convex subset of $X$. Then $D$ is homotopy dense in $X$.

The following lemma is an immediate consequence of [CDM93, Lemma 8.10] and the previous lemma.

Lemma 4.3. Let $X$ be a Polish convex subset of a Fréchet space and $D$ be a dense convex subset of $X$ contained in a $\sigma Z$-set $Y \subset X$. Then for any compact metrizable space $K$ and a $\sigma$-compact subset $A \subset K$ there exists a continuous map $f: K \rightarrow X$ such that $f(A) \subset D$ and $f(K \backslash A) \subset X \backslash Y$.

A subset $C$ of a linear topological space $L$ is called $\infty$-convex if for any bounded sequence $\left(x_{n}\right)_{n \geq 0}$ in $C$ and any sequence of nonnegative reals $t_{n}$ with $\sum_{n>0} t_{n}=1$ the series $\sum_{n \geq 0} t_{n} x_{n}$ converges to some point of $C$. Recall that a subset $B$ of $L$ is bounded if for every neighborhood $U \subset L$ of zero $B$ is contained in all but finitely many sets $n U$, $n \in \mathbb{N}$.

Recall that a subspace $A$ of a Polish space $B$ is Polish if and only if $A$ is $G_{\delta}$ in $B$. 
THEOREM 4.4. For an integer $l \geq 0$ let $C_{\bullet}^{l}$ be an $\infty$-convex $G_{\delta}$ subset of $C^{l}(V)$. For $\gamma \in[l, \infty]$ set $C_{\bullet}^{\gamma}=C_{\bullet}^{l} \cap C^{\gamma}(V)$. If $n \geq l$ is an integer such that for every integer $k \geq n$ the subset $C_{\bullet}^{\infty}$ is dense in $C_{\bullet}^{k}$, and $C_{\bullet}^{\bar{k}+1}$ is contained in a $\sigma Z$-subset of $C_{\bullet}^{k}$, then $\left(C_{\bullet}^{n}, C_{\bullet}^{\infty}\right)$ is $\left(\mathcal{M}_{0}, \mathcal{M}_{2}\right)$-preuniversal.

Proof. We shall apply the technique used in the proof of [Ban00, Theorem 5.1, p.73]. Fix $n \geq l$ and a pair $(K, M) \in\left(\mathcal{M}_{0}, \mathcal{M}_{2}\right)$. We wish to construct a continuous map $f: K \rightarrow C_{\bullet}^{n}$ such that $f(M) \subset C_{\bullet}^{\infty}$ and $f(K \backslash M) \subset C_{\bullet}^{n} \backslash C_{\bullet}^{\infty}$.

Since $C_{\bullet}^{l}$ is $G_{\delta}$ in $C^{l}(V)$, for each integer $k \geq l$ the subset $C_{\bullet}^{k}$ is $G_{\delta}$ in $C^{k}(V)$, and in particular $C_{\bullet}^{k}$ is Polish. Since $M \in \mathcal{M}_{2}$ we can write $M=\bigcap_{k=n}^{\infty} A_{k}$ where $\left(A_{k}\right)_{k=n}^{\infty}$ is a decreasing sequence of $\sigma$-compact subsets of $K$. By Lemma 4.3 for each $k \geq n$ there exists a continuous map $f_{k}: K \rightarrow C_{\bullet}^{k}$ such that $f_{k}\left(A_{k}\right) \subset C_{\bullet}^{\infty}$ and $f_{k}\left(K \backslash A_{k}\right) \cap C^{k+1}(V)=\emptyset$.

Fix a basepoint $b \in C_{\bullet}^{\infty}$; we also think of $b$ as a constant map taking $K$ to $\{b\}$. Replacing $f_{k}$ by $\left(1-\varepsilon_{k}\right) b+\varepsilon_{k} f_{k}$ for a sufficiently small positive $\varepsilon_{k}$ we can additionally assume that

$$
f_{k}(K) \subset\left\{g \in C_{\bullet}^{k}:\|g-b\|_{C^{k}\left(D_{k}\right)} \leq 2^{-k}\right\} .
$$

Let us show that the series

$$
\sum_{k \geq n} 2^{-k}\left(f_{k}(z)-b\right)
$$

converges uniformly in $C^{n}(V)$ where $z \in K$. To this end we give $C^{n}(V)$ the metric $\sum_{j \geq n}\left(p_{j} /\left(p_{j}+1\right)\right) 2^{-j}$, where $p_{j}=\|\cdot\|_{C^{n}\left(D_{j}\right)}$, and check that the sequence of partial sums of the series (4.6) is Cauchy. Fix $j$, suppose $i>m>\max (n, j)$, and estimate

$$
\left\|\sum_{k=m}^{i} 2^{-k}\left(f_{k}(z)-b\right)\right\|_{C^{n}\left(D_{j}\right)} \leq \sum_{k=m}^{i} 2^{-k}\left\|f_{k}(z)-b\right\|_{C^{k}\left(D_{k}\right)} \leq 2^{-m} \sum_{k=m}^{i} 2^{-k}<2^{-m}
$$

where in the first inequality we used the definition (4.1) and the fact that $D_{j} \subset D_{k}$ for all $j<k$, while the second inequality depends on (4.5). This proves uniform convergence of (4.6) and therefore defines a continuous map $f: K \rightarrow C^{n}(V)$ given by

$$
f=b+2^{n-1} \cdot \sum_{k \geq n} 2^{-k}\left(f_{k}-b\right)=2^{n-1} \cdot \sum_{k \geq n} 2^{-k} f_{k} .
$$

Next we show that for every $z \in K$ the sequence $\left(f_{k}(z)\right)_{k \geq l}$ is bounded in $C^{n}(V)$. Note first that

$$
\left\|f_{k}(z)-b\right\|_{C^{l}\left(D_{l}\right)} \leq\left\|f_{k}(z)-b\right\|_{C^{k}\left(D_{k}\right)} \leq 2^{-k} \leq 2^{-l}
$$

so that

$$
\left\|f_{k}(z)\right\|_{C^{l}\left(D_{l}\right)} \leq 2^{-l}+\|b\|_{C^{l}\left(D_{l}\right)}
$$

and since any neighborhood of 0 in $C^{n}(V)$ contains a neighborhood of the form 
$\left\{g \in C^{n}(V):\|g\|_{C^{l}\left(D_{l}\right)}<\varepsilon\right\}$, the sequence $\left(f_{k}(z)\right)_{k \geq l}$ is bounded in $C^{n}(V)$.

The $\infty$-convexity of $C_{\bullet}^{l}$ in $C^{l}(V)$ implies that $C_{\bullet}^{\gamma}$ is $\infty$-convex in $C^{\gamma}(V)$ for every $\gamma \in[l, \infty]$. Since $f_{k}(z) \in C_{\bullet}^{n}$ for each $z \in K$ and $k \geq n$, the right hand side of (4.7) lies in $C_{\bullet}^{n}$, i.e., $f(K) \subset C_{\bullet}^{n}$. By the same token $f(M) \subset C_{\bullet}^{\infty}$.

It remains to prove that $f(z) \notin C^{\infty}(V)$ for every $z \in K \backslash M$. Fix such $z$ and find a unique $m>n$ with $z \in A_{m-1} \backslash A_{m}$. Thus $z \in A_{k}$ for $k<m$ and $z \notin A_{k}$ for $k \geq m$. The choice of $z$ and $f_{k}$ guarantee that

- $f_{m}(z) \in C^{m}(V) \backslash C^{m+1}(V)$,

- $f_{k}(z) \in C^{\infty}(V)$ for all $k<m$,

- $f_{k}(z) \in C^{m+1}(V)$ for all $k>m$.

In the right hand side of

$$
2^{1-n} f(z)=2^{-m} f_{m}(z)+\sum_{k=n}^{m-1} 2^{-k} f_{k}(z)+\sum_{k>m} 2^{-k} f_{k}(z)
$$

the second summand is in $C^{\infty}(V)$ and the third summand converges in the Fréchet space $C^{m+1}(V)$. Since $f_{m}(z)$ is not in $C^{m+1}(V)$ neither is $f(z)$.

Lemma 4.8. If $(A, B)$ is a $\left(\mathcal{M}_{0}, \mathcal{M}_{2}\right)$-preuniversal pair, and $f: A \rightarrow X$ is a continuous injective map to a Hausdorff topological space $X$, then the subspace $f(B)$ of $X$ is $\mathcal{M}_{2}$-universal and the pair $(X, f(B))$ is $\left(\mathcal{M}_{0}, \mathcal{M}_{2}\right)$-universal.

Proof. The pair $(A, B)$ is $\left(\mathcal{M}_{0}, \mathcal{M}_{2}\right)$-universal by the above-mentioned equivalence of universality and preuniversality. Thus for each pair $(K, M) \in\left(\mathcal{M}_{0}, \mathcal{M}_{2}\right)$ there is a topological embedding $g: K \rightarrow A$ such that $g^{-1}(B)=M$. Since $K$ is compact and $X$ is Hausdorff, the continuous injective map $h=f \circ g: K \rightarrow X$ is a topological embedding. The injectivity of $f$ guarantees that $h^{-1}(f(B))=g^{-1}(B)=M$. Thus the map $h$ witnesses $\left(\mathcal{M}_{0}, \mathcal{M}_{2}\right)$-universality of the pair $(X, f(B))$. We can choose the pair $(K, M)$ so that $M$ is $\mathcal{M}_{2}$-universal (e.g., using that each separable metric space embeds into the Hilbert cube). The homeomorphism $h: K \rightarrow h(K)$ restricts to a homeomorphism $M \rightarrow h(M)=f(B)$, so the latter is $\mathcal{M}_{2}$-universal.

Theorem 4.4 and Lemma 4.8 immediately imply the following corollary, which is the main result of this section.

Corollary 4.9. Let $\left(C_{\bullet}^{n}, C_{\bullet}^{\infty}\right)$ be as in Theorem 4.4. If $f: C_{\bullet}^{n} \rightarrow X$ is a continuous injective map to a Hausdorff topological space $X$, then the subspace $f\left(C_{\bullet}^{\infty}\right)$ of $X$ is $\mathcal{M}_{2}$-universal.

\section{5. $\mathcal{M}_{2}$-universal spaces of smooth functions.}

In this section we give a construction of sequences $\left(C_{\bullet}^{n}, C_{\bullet}^{\infty}\right)_{n \geq l}$ satisfying the assumptions of Theorem 4.4 which covers all examples arising in the present paper, and also give a criterion of when a space of smooth maps belongs to the class $\mathcal{M}_{2}$. 
Let $N$ be a smooth manifold, possibly with boundary. With the $C^{m}$ topology the space $C^{m}(N)$ of $m$-differentiable functions on $N$ is a separable Fréchet space, where $m$ is a nonnegative integer or $\infty$, see Section 2 .

THEOREM 5.1. Let $N$ be a smooth manifold, possibly with boundary, and let $D \subset$ Int $N$ be a smoothly embedded top-dimensional closed disk that is mapped via a coordinate chart to a Euclidean unit disk. Let $l \geq 0$ be an integer and let $\mathfrak{D}: C^{l}(N) \rightarrow C^{0}(N)$ be a continuous linear map. Given $\eta \in \mathbb{R}$ suppose there exists $h_{\bullet} \in C^{\infty}(N)$ with $\left.\mathfrak{D} h_{\bullet}\right|_{D}>\eta$. Let $C_{\bullet}^{l}$ denote the subspace of $C^{l}(N)$ of functions $u$ such that $\left.\mathfrak{D} u\right|_{D} \geq \eta$ and $\left.u\right|_{N \backslash \operatorname{Int} D}=$ $h_{\bullet}$ Then for $C_{\bullet}^{n}=C_{\bullet}^{l} \cap C^{n}(N)$ and $C_{\bullet}^{\infty}=C_{\bullet}^{l} \cap C^{\infty}(N)$ the sequence $\left(C_{\bullet}^{n}, C_{\bullet}^{\infty}\right)_{n \geq l}$ satisfies the assumptions of Theorem 4.4 .

Proof. For every $n \geq l$ the subset $C_{\bullet}^{n}$ is clearly convex and closed in the Fréchet space $C^{n}(N)$, so in particular, $C_{\bullet}^{n}$ is $G_{\delta}$ in $C^{n}(N)$.

The $\infty$-convexity of $C_{\bullet}^{l}$ follows from the fact that any closed convex subset of a Fréchet space is $\infty$-convex. (To prove the fact suppose $x_{n}, t_{n}, C, L$ are as in the definition of $\infty$-convexity given before Theorem 4.4, and the Fréchet structure on $L$ is given by a countable family of seminorms $p_{k}$. Since $\left\{x_{n}\right\}$ is bounded, $p_{k}\left(x_{n}\right)$ is bounded by a constant depending only on $k$, which easily implies that the partial sums of the series $\sum_{n>0} t_{n} x_{n}$ form a Cauchy sequence in $L$. Their limit in $L$ is also the limit of the sequence $\left(\sum_{n=0}^{m} t_{n}\right)^{-1} \sum_{n=0}^{m} t_{n} x_{n}$ which lies in $\left.C\right)$.

For a positive integer $m$ and a point $p \in D$ let $Z_{m}$ be the set of all $u \in C_{\bullet}^{n+1}$ whose $C^{k}(p)$ norm is $\leq m$ (the norm is simply the sum of the absolute values of all partial derivatives of $u$ of orders $\leq k$ evaluated at $p$ ). It is easy to check that $Z_{m}$ is a $Z$-set, see the proof of Lemma 12.1, and clearly $C_{\bullet}^{n+1}$ equals in the union of the sets $Z_{m}$.

To show that $C_{\bullet}^{\infty}$ is dense in $C_{\bullet}^{n}$ fix $u \in C_{\bullet}^{n}$. Replacing $u$ with $\delta h_{\bullet}+(1-\delta) u$, where $\delta$ is small and positive, we can assume that $\left.\mathfrak{D} u\right|_{D}>\eta$. Henceforth we suppress distinctions between the objects in the Euclidean space and their images under the coordinate chart containing $D$. In the chart we fix a concentric closed disk $D_{\varepsilon} \subset \operatorname{Int} D$ such that $D$, $D_{\varepsilon}$ have radii $1,1-\varepsilon$, respectively. Consider the partition of unity $\left\{\psi_{\varepsilon}, 1-\psi_{\varepsilon}\right\}$ such that $\left.\psi_{\varepsilon}\right|_{D_{\varepsilon}}=1$, the support of $\psi_{\varepsilon}$ equals $D$, and $\psi_{\varepsilon}$ is rotationally symmetric. We also assume that the $k$-th radial derivative of $\psi_{\varepsilon}$ grows with $\varepsilon$ as $\varepsilon^{-k}$ which can be arranged as follows: Start with a smooth function $\psi: \mathbb{R} \rightarrow[0,1]$ with $\left.\psi\right|_{[-\infty, 1]}=1$ and $\left.\psi\right|_{[2, \infty]}=0$, and set $\psi_{\varepsilon}(r)=\psi(2-(1-r) / \varepsilon)$ for $r \geq 0$.

Approximate $\left.u\right|_{D}$ by a family $\left\{u_{\tau}\right\}_{\tau \geq 0}$ of smooth maps such that $\left.u_{\tau} \rightarrow u\right|_{D}$ in $C^{n}(D)$ as $\tau \rightarrow 0$. Then glue $u_{\tau}$ and $h_{\bullet}$ via the partition of unity $\left\{\psi_{\varepsilon}, 1-\psi_{\varepsilon}\right\}$. The result $\left(1-\psi_{\varepsilon}\right) h_{\bullet}+\psi_{\varepsilon} u_{\tau} \in C^{\infty}(N)$ equals $u_{\tau}$ on $D_{\varepsilon}$ and $h_{\bullet}$ on $M \backslash$ Int $D$.

We are going to show that $\left(1-\psi_{\varepsilon}\right) h_{\bullet}+\psi_{\varepsilon} u_{\tau}$ approximates $u$ in $C^{n}(N)$ for suitable $\varepsilon, \tau$. Since $\left.\mathfrak{D} u\right|_{D}>\eta$ this would imply $\left(1-\psi_{\varepsilon}\right) h_{\bullet}+\psi_{\varepsilon} u_{\tau} \in C_{\bullet}^{\infty}$. Write

$$
\left(1-\psi_{\varepsilon}\right) h_{\bullet}+\psi_{\varepsilon} u_{\tau}-u=\left(1-\psi_{\varepsilon}\right)\left(h_{\bullet}-u\right)+\psi_{\varepsilon}\left(u_{\tau}-u\right)
$$

On the right hand side for every fixed $\varepsilon$ the second summand converges to zero in $C^{n}(D)$ as $\tau \rightarrow 0$, hence it suffices to show that the first summand converges to zero in $C^{n}(D)$ as $\varepsilon \rightarrow 0$. 
Set $v=h_{\bullet}-u$. Note that $v \in C^{n}(N)$ and $v$ vanishes on $M \backslash$ Int $D$; in particular, all derivatives of $v$ of orders $\leq n$ vanish on $\partial D$. Arguing by contradiction suppose that there is a sequence of points of $D$ where the derivatives of $v\left(1-\psi_{\varepsilon}\right)$ of some order $k \leq n$ do not approach zero as $\varepsilon \rightarrow 0$. By compactness we may assume that the points converge to a point $p$, which has to lie on $\partial D$. Introduce new coordinates $(r, \theta)$ near $p$ where $r$ is the distance to $\partial D$ and $\theta$ represents the remaining coordinates along $\partial D$. Since $\psi_{\varepsilon}$ does not depend on $\theta$, the only derivatives of $v\left(1-\psi_{\varepsilon}\right)$ that might not approach zero are the derivatives by $r$. Hence we need to analyze the terms $\left(1-\psi_{\varepsilon}\right)^{(k-m)}\left(\partial^{m} v / \partial r^{m}\right)$ for $0 \leq m<k$; the case $m=k$ is trivial since $1-\psi_{\varepsilon}$ is bounded independently of $\varepsilon$. Consider the order $k-m$ Taylor expansion of $\partial^{m} v / \partial r^{m}$ at $p$ with respect to $r$. The only (possibly) nonzero term is the remainder $\left(r^{k-m} /(k-m) !\right)\left(\partial^{k} v / \partial r^{k}\right)(\xi, \theta)$ where $0<\xi<r$. We can restrict attention to $r<\varepsilon$ because $1-\psi_{\varepsilon}$ vanishes for $r \geq \varepsilon$. By the choice of $\psi_{\varepsilon}$ the term $\left|\left(1-\psi_{\varepsilon}\right)^{(k-m)}\right| \varepsilon^{k-m}$ is bounded, so there is a constant $C>0$ such that

$$
\left|\left(1-\psi_{\varepsilon}\right)^{(k-m)} \frac{\partial^{m} v}{\partial r^{m}}\right| \leq\left|\left(1-\psi_{\varepsilon}\right)^{(k-m)} \frac{\partial^{k} v}{\partial r^{k}}(\xi, \theta) \frac{\varepsilon^{k-m}}{(k-m) !}\right| \leq C\left|\frac{\partial^{k} v}{\partial r^{k}}(\xi, \theta)\right|
$$

where the right hand side approaches zero as $\varepsilon \rightarrow 0$ because $0<\xi<\varepsilon$. This contradiction completes the proof that $C_{\bullet}^{\infty}$ is dense in $C_{\bullet}^{n}$.

Let $\Gamma^{\gamma}\left(V, \mathbb{R}^{n}\right)$ denotes the space of smooth maps from a manifold $V$ to $\mathbb{R}^{n}$ endowed with the $C^{\gamma}$ topology, $\gamma \in[0, \infty]$. Recall that $\mathcal{M}_{2}$ is the class of spaces homeomorphic to $F_{\sigma \delta}$-sets in metrizable compacta.

LEMma 5.2. For every $\gamma$ the identity map id: $\Gamma^{\infty}\left(V, \mathbb{R}^{n}\right) \rightarrow \Gamma^{\gamma}\left(V, \mathbb{R}^{n}\right)$ takes any $G_{\delta}$ subset to a space in $\mathcal{M}_{2}$.

Proof. Fix a $G_{\delta}$ subset $S^{\infty}$ of $\Gamma^{\infty}\left(V, \mathbb{R}^{n}\right)$ and set $S^{\gamma}=\operatorname{id}\left(S^{\infty}\right)$. Recall that a (not necessarily continuous) map is $F_{\sigma}$-measurable if the preimage of any open set is $F_{\sigma}$. To prove the lemma it suffices to show that the inverse of the identity map id: $S^{\infty} \rightarrow S^{\gamma}$ is $F_{\sigma}$-measurable because in this case the fact that id is continuous (and hence $F_{\sigma}$-measurable) implies that the map id is a $(1,1)$ homeomorphism in the terminology of [Kur66, Corollary 3 in VII, section 35], where it is shown that any $(1,1)$ homeomorphism maps a completely metrizable separable space (such as $S^{\infty}$ ) to a space in $\mathcal{M}_{2}$.

Since the restriction and the enlarging of a co-domain of an $F_{\sigma}$-measurable map is $F_{\sigma}$-measurable, it is enough to show that the inverse of the identity map id: $\Gamma^{\infty}\left(V, \mathbb{R}^{n}\right) \rightarrow$ $\Gamma^{\gamma}\left(V, \mathbb{R}^{n}\right)$ is $F_{\sigma}$-measurable.

If $Y$ is separable and metrizable, then to prove that a map $f: X \rightarrow Y$ is $F_{\sigma}$-measurable it (clearly) suffices to show that for each open set $W \subset Y$ and each $y \in W$ there is a subset $T_{y} \subset W$ with $y \in \operatorname{Int} T_{y}$ such that $f^{-1}\left(T_{y}\right)$ is closed. Let us apply this to $f=\mathrm{id}: \Gamma^{\gamma}\left(V, \mathbb{R}^{n}\right) \rightarrow \Gamma^{\infty}\left(V, \mathbb{R}^{n}\right)$.

Fix an exhaustion of $V$ by compact subsets $D_{j}$, fix some norm on $\mathbb{R}^{n}$, and denote by $\|\cdot\|_{C^{m}\left(D_{j}\right)}$ the associated $C^{m}$ norm. Given any $m>\gamma$ note that

$$
T_{y}=y+\left\{f \in \Gamma^{\gamma}\left(V, \mathbb{R}^{n}\right):\|f\|_{C^{m}\left(D_{j}\right)} \leq \varepsilon\right\}
$$


is closed in $\Gamma^{\gamma}\left(V, \mathbb{R}^{n}\right)$ and $y \in \operatorname{Int} T_{y}$. Also $T_{y}$ lies inside any neighborhood of $y$ in $\Gamma^{\infty}\left(V, \mathbb{R}^{n}\right)$ if $\varepsilon$ is small enough.

\section{Space of all complete Riemannian metrics: smooth topology.}

In this section we prove Theorem 1.1 for $\gamma=\infty$. Let $V$ be a smooth connected manifold of positive dimension. The space $\mathcal{R}^{\gamma}(V)$ of all complete smooth Riemannian metrics on a manifold $V$ is a convex subset in the metric linear space $\mathcal{T}^{\gamma}(V)$ of smooth symmetric 2-tensors on $V$ with the $C^{\gamma}$ topology.

Lemma 6.1. $\quad \mathcal{R}^{\gamma}(V)$ is a $G_{\delta}$ subset of $\mathcal{T}^{\gamma}(V)$.

Proof. Let $\left(K_{n}\right)$ be a countable family of compact subsets exhausting $V$. Then $\mathcal{R}^{\gamma}(V)$ is the intersection of the the sets $\left\{t \in \mathcal{T}^{\gamma}(V):\left.t\right|_{K_{n}}\right.$ is positive definite $\}$ which are open in $\mathcal{T}^{\gamma}(V)$.

REMARK 6.2. $\quad \mathcal{R}^{\gamma}(V)$ is (clearly) open if $V$ is compact, and has empty interior if $V$ is noncompact, see [FM78].

Note that $\mathcal{T}^{\infty}(V)$ is Fréchet. To show that $\mathcal{R}^{\infty}(V)$ is homeomorphic to $\ell^{2}$ it is enough to check that the closure of $\mathcal{R}^{\infty}(V)$ in $\mathcal{T}^{\infty}(V)$ is not locally compact [DT81, Theorem 2]. The closure is the convex set $\mathcal{N}^{\infty}(V)$ of smooth symmetric non-negative definite 2-tensors.

LEMma 6.3. $\quad \mathcal{N}^{\infty}(V)$ is not locally compact.

Proof. This is a variation of [BH15, Lemma 2.5]. Suppose arguing by contradiction that every point of $\mathcal{N}^{\infty}(V)$ has a compact neighborhood and let $K$ be a compact neighborhood of $g \in \mathcal{R}^{\infty}(V)$ in $\mathcal{N}^{\infty}(V)$. Fix a disk $D \subset V$ inside a coordinate chart, and consider the restriction map $\delta: \mathcal{N}^{\infty}(V) \rightarrow \mathcal{T}^{\infty}(D)$. Continuity of $\delta$ implies that $\delta(K)$ is compact.

Let us show that $\delta(K)$ is also a neighborhood of $\delta(g)$. If not, then there is a sequence $\check{\tau}_{i} \notin \delta(K)$ converging to $\delta(g)$ in $\mathcal{T}^{\infty}(D)$. Extend $\check{\tau}_{i}$ to $\tau_{i} \in \mathcal{T}^{\infty}(V)$ converging to $g$. Let $\phi$ a bump function on $V$ with $\left.\phi\right|_{D}=1$. Then $g+\phi\left(\tau_{i}-g\right)$ converges to $g$ and hence lies in $K$ for large $i$. The restriction of $g+\phi\left(\tau_{i}-g\right)$ to $D$ equals $\check{\tau}_{i}$ so $\check{\tau}_{i} \in \delta(K)$, which is a contradiction.

Thus $\delta(g)$ has a compact neighborhood in $\mathcal{T}^{\infty}(D)$, which is a Fréchet space. Hence any point in the Fréchet space has a compact neighborhood, which implies that $\mathcal{T}^{\infty}(D)$ is finite dimensional [Trè67, Theorem 9.2], which is clearly false. This contradiction proves that $\mathcal{N}^{\infty}(V)$ is not locally compact.

\section{Space of all complete Riemannian metrics: $C^{\gamma}$ topology.}

In this section we prove Theorem 1.1 for finite $\gamma$.

LEMMA 7.1. If $\gamma$ is finite, then $\mathcal{R}^{\gamma}(V)$ is $\sigma Z$. 
ProOF. With minor modifications the argument of Lemma 12.1 works for $\mathcal{R}^{\gamma}(V)$, namely we choose $\alpha$ to take values in the space of nonnegative definite 2-tensors on $V$ which ensures that $f(q)+\alpha \in \mathcal{R}^{\gamma}(V)$ for all $q$.

LEMMA 7.2. $\mathcal{R}^{\gamma}(V)$ belongs to $\mathcal{M}_{2}$.

Proof. By Lemma 6.1 the subspace $\mathcal{R}^{\infty}(V)$ is $G_{\delta}$ in the linear space of all smooth 2-tensors on $V$ with $C^{\infty}$ topology, so $\mathcal{R}^{\gamma}(V)$ is completely metrizable so that Lemma 5.2 applies.

Lemma 7.3. $\quad \mathcal{R}^{\gamma}(V)$ is $\mathcal{M}_{2}$-universal for every finite $\gamma$.

Proof. It is enough to show that $\mathcal{R}^{\gamma}(V)$ contains a closed $\mathcal{M}_{2}$-universal subspace. Fix any integer $l \geq \gamma$ and let $\mathfrak{D}: C^{l}(V) \rightarrow C^{0}(V)$ be the inclusion. Let $h_{\bullet} \in C^{\infty}(V)$ be the constant function that equals 2 at all points, let $\eta=1$, and let $D$ be an embedded coordinate disk in $V$. Let $C_{\bullet}^{l}$ be the set of functions $u \in C^{l}(V)$ with such that $\left.u\right|_{D} \geq \eta$ and $\left.u\right|_{V \backslash \text { Int } D}=h_{\bullet}$. Let $X$ be the space of all metrically complete $C^{l}$-Riemannian metrics on $V$ with the $C^{\gamma}$ topology. By Theorem 5.1 and Corollary 4.9 for any continuous injective map from $f: C_{\bullet}^{l} \rightarrow X$ the space $f\left(C^{\infty}(V) \cap C_{\bullet}^{l}\right)$ is $\mathcal{M}_{2}$-universal.

To define $f$ fix $g \in \mathcal{R}^{\infty}(V)$ and let $f(u)=u g$. The map $f$ is continuous as $l \geq \gamma$ and injective because $g$ is positive definite. Each metric in $f\left(C^{\infty}(V) \cap C_{\bullet}^{l}\right)$ is smooth because $u$ is smooth, and complete since $u g=2 g$ outside $D$ and $2 g$ is complete.

It remains to show that $f\left(C^{\infty}(V) \cap C_{\bullet}^{l}\right)$ is closed in $\mathcal{R}^{\gamma}(V)$. To this end suppose $u_{i} g \in f\left(C^{\infty}(V) \cap C_{\bullet}^{l}\right)$ converges in $C^{\gamma}$ topology to a smooth complete metric $g_{*}$. Thus $g_{*}=2 g$ outside $D$. Choose a $g$-unit vector field $U$ on a neighborhood $W$ of $D$. In the $C^{\gamma}$ topology on $W$ the functions $u_{i}=u_{i} g(U, U)$ converge to $g_{*}(U, U)$, which equals 2 on $W \backslash D$. Let $u$ be a function that equals $g_{*}(U, U)$ on $W$ and equals 2 outside $D$; clearly $u$ is smooth. Hence the metrics $u_{i} g$ converge to $u g$ in the $C^{\gamma}$ topology on $V$. By uniqueness of the limit $g_{*}=u g$.

REMARK 7.4. The same proofs show that the space of all (complete or not) smooth Riemannian metrics on a manifold $V$ equipped with the $C^{\gamma}$ topology is homeomorphic to $\ell^{2}$ when $\gamma=\infty$ and $\mathcal{M}_{2}$-universal if $\gamma$ is finite.

TheOrem 7.5. $\mathcal{R}^{\gamma}(V)$ is $\mathcal{M}_{2}$-absorbing, and hence is homeomorphic to $\Sigma^{\omega}$.

Proof. Let $\overline{\mathcal{T}}^{\gamma}(V)$ be the closure of $\mathcal{T}^{\gamma}(V)$ of Section 6 in the Fréchet space of all $C^{\gamma}$ 2-tensors on $V$ with the $C^{\gamma}$ topology; thus $\overline{\mathcal{T}}^{\gamma}(V)$ is a separable Fréchet space. The closure of $\mathcal{R}^{\gamma}(V)$ in $\overline{\mathcal{T}}^{\gamma}(V)$ is not contained in Aff $\mathcal{R}^{\gamma}(V)$ because it clearly contains some non-smooth metrics. Hence by Lemma $3.1 \mathcal{R}^{\gamma}(V)$ is homeomorphic to a homotopy dense subset of $\ell^{2}$. The proof of Lemma 7.3 actually shows that the $\mathcal{M}_{2}$-universal subset $f\left(C^{\infty}(V) \cap C_{\bullet}^{l}\right)$ is closed in $\mathcal{T}^{\gamma}(V)$. Therefore [BRZ96, Proposition 5.3.5] implies that $\mathcal{R}^{\gamma}(V)$ is strongly $\mathcal{M}_{2}$-universal. Thus Lemmas 7.1 and 7.2 imply that $\mathcal{R}^{\gamma}(V)$ is $\mathcal{M}_{2^{-}}$ absorbing. Since $\mathcal{R}^{\gamma}(V)$ is contractible, it is homeomorphic to $\Sigma^{\omega}$. 


\section{Diffeomorphism groups and uniformization of $S^{2}$ and $R P^{2}$.}

Let $g_{1}$ denote the standard curvature 1 metric on $S^{2}$ and $R P^{2}$, the unit sphere and its quotient by the antipodal map. Henceforth we identify $S^{2}$ with $\overline{\mathbb{C}}$ so that the antipodal map corresponds to $z \rightarrow-\bar{z}^{-1}$, and the group of orientation-preserving conformal transformation of $\left(S^{2}, g_{1}\right)$ corresponds to $\operatorname{PSL}(2, \mathbb{C})$, which acts simply transitively on triples of distinct points of $S^{2}$. Fix any triple of distinct points of $S^{2}$, and let $D^{\beta}\left(S^{2}\right)$ be the group of smooth orientation-preserving diffeomorphisms of $S^{2}$ fixing the triple and equipped with the $C^{\beta}$ topology, $\beta \in[1, \infty]$.

A diffeomorphism of $R P^{2}$ has two lifts to the orientation cover: a lift homotopic to the identity of $S^{2}$ and its composite with the antipodal map. So any diffeomorphism of $R P^{2}$ lifts to a unique orientation-preserving diffeomorphism of $S^{2}$ which by uniqueness must commute with the antipodal map. Since lifts of conformal diffeomorphism are conformal, the orientation-preserving lifts of conformal diffeomorphisms of $\left(R P^{2}, g_{1}\right)$ are precisely the elements of $P S L(2, \mathbb{C})$ commuting with the antipodal map. It is straightforward to check that these lifts form the subgroup $P S U(2) \subset P S L(2, \mathbb{C})$, which corresponds under the above identification to $S O(3)$ acting on the unit sphere, and which acts simply transitively on the unit tangent bundle to $R P^{2}$. Fix a vector in the unit tangent bundle of $R P^{2}$ and let $D^{\beta}\left(R P^{2}\right)$ be the group of all diffeomorphisms of $R P^{2}$ that fix the vector.

If $M$ is $S^{2}$ or $R P^{2}$, then by the Uniformization Theorem any metric on $M$ is of the form $\phi^{*} e^{-2 u} g_{1}$ where $u \in C^{\infty}(M)$ and $\phi$ is a diffeomorphism of $M$. (The standard accounts such as e.g. [Don11, Chapter 10] omit the case of $R P^{2}$ which we include for completeness. Let $\left(S^{2}, \phi^{*} e^{-2 u} g_{1}\right)$ be the pullback of a given metric $\left(R P^{2}, g\right)$ to the orientation cover. The covering isometry of $\phi^{*} e^{-2 u} g_{1}$ gives rise to an isometric involution $\iota$ of $e^{-2 u} g_{1}$. Hence $\iota^{*} g_{1}=e^{2(u \circ \iota-u)} g_{1}$, and since the left hand side has a transitive isometry group $u \circ \iota-u$ is constant, which we denote $\lambda$. Precomposing $u \circ \iota-u=\lambda$ with $\iota$ gives $u-u \circ \iota=\lambda$, so $\lambda=0$. Hence $\iota$ is an isometry of $g_{1}$ and $u$ is $\iota$-invariant. It follows that $e^{-2 u} g_{1}$ descends to a metric on $R P^{2}$ of desired form).

It is routine to check that $D^{\beta}(M)$ is a topological group. (For $\beta \notin \mathbb{Z}$ the relevant properties of Hölder map and inverses of Hölder diffeomorphisms can be found, e.g., in [BHS05, Section 2.2]).

Thus if $M$ equals $S^{2}$ or $R P^{2}$ then no nontrivial element of $D^{\beta}(M)$ is conformal, and any orientation-preserving diffeomorphism of $M$ can be written uniquely as the composite of an element of $D^{\beta}(M)$ followed by a conformal automorphism.

For $\gamma \in[0, \infty]$ let $\Gamma^{\gamma}(M)$ denote the linear space of all smooth functions on $M$ with the $C^{\gamma}$ topology; thus as a set $\Gamma^{\gamma}(M)$ equals $C^{\infty}(M)$.

For $M$ equal to $S^{2}$ or $R P^{2}$ the above discussion shows that the map

$$
D^{\gamma+1}(M) \times \Gamma^{\gamma}(M) \rightarrow \mathcal{R}^{\gamma}(M)
$$

given by $(\phi, u) \rightarrow \phi^{*} e^{-2 u} g_{1}$ is a continuous bijection.

The sectional curvature of $e^{-2 u} g_{1}$ equals $e^{2 u}\left(1+\triangle_{g_{1}} u\right)$ where $\Delta_{g_{1}}$ is the $g_{1}$-Laplacian, see [KW74, p.15, (1.3)].

Let $\mathcal{O}_{\geq \lambda}^{\gamma}(M) \subset \Gamma^{\gamma}(M)$ be the subset of functions $u$ such that $e^{-2 u} g_{1}$ has sectional 
curvature $\geq \lambda$; the subset $\mathcal{O}_{>\lambda}^{\gamma}(M)$ is defined similarly.

LEMmA 8.2. The subsets $\mathcal{O}_{\geq \lambda}^{\gamma}(M)$ and $\mathcal{O}_{>\lambda}^{\gamma}(M)$ are convex in $\Gamma^{\gamma}(M)$.

Proof. Let $u=t u_{1}+(1-t) u_{0}$ where $t \in[0,1]$ and $u_{0}, u_{1} \in \mathcal{O}_{\geq \lambda}^{\gamma}(M)$. Then $1+\triangle_{g_{1}} u=t\left(1+\triangle_{g_{1}} u_{1}\right)+(1-t)\left(1+\triangle_{g_{1}} u_{0}\right) \geq \lambda\left(t e^{-2 u_{1}}+(1-t) e^{-2 u_{0}}\right) \geq \lambda e^{-2 u}$

where the last inequality holds by convexity of the exponential function. Thus the sectional curvature of $e^{-2 u} g_{1}$ is $e^{2 u}\left(1+\triangle_{g_{1}} u\right) \geq \lambda$.

The map (8.1) restricts to the continuous bijections

$$
D^{\gamma+1}(M) \times \mathcal{O}_{\geq \lambda}^{\gamma}(M) \rightarrow \mathcal{R}_{\geq \lambda}^{\gamma}(M) \quad D^{\gamma+1}(M) \times \mathcal{O}_{>\lambda}^{\gamma}(M) \rightarrow \mathcal{R}_{>\lambda}^{\gamma}(M) .
$$

TheOREM 8.4. If $\gamma \notin \mathbb{Z}$, then the maps (8.1) and (8.3) are homeomorphisms, and in particular, $D^{\gamma+1}(M), \mathcal{R}_{\geq \lambda}^{\gamma}(M), \mathcal{R}_{>\lambda}^{\gamma}(M)$ are contractible.

Proof. This is a minor modification of [BH15, Theorem 4.1] whose statement incorrectly assumes $\gamma \in \mathbb{Z}$ instead of $\gamma \notin \mathbb{Z}$, cf. [BH16]. The proof is the same and the main ingredient is that the solution of Beltrami equation in a chart depends in Hölder topology on the dilatation, which is where $\gamma \notin \mathbb{Z}$ is used. Relevant properties of Hölder map can be found, e.g., in [BHS05, Section 2.2]. Since $D^{\gamma+1}(M)$ is a retract of the convex set $\mathcal{R}^{\gamma}(M)$, it is contractible. This together with convexity of $\mathcal{O}_{\geq \lambda}^{\gamma}(M)$ and $\mathcal{O}_{>\lambda}^{\gamma}(M)$ implies contractibility of $\mathcal{R}_{\geq \lambda}^{\gamma}(M)$ and $\mathcal{R}_{>\lambda}^{\gamma}(M)$.

REMARK 8.5. S. Smale proved in [Sma59] that $D^{\beta}\left(S^{2}\right)$ is contractible for every integer $\beta>1$. The same should be true for $\beta=1$, as well as for $D^{\beta}\left(R P^{2}\right)$ where $\beta$ is a positive integer, but we have not checked the details and are not aware of any proof in the literature.

Corollary 8.6. Let $M$ be $S^{2}$ or $R P^{2}$. Then $D^{\infty}(M)$ is homeomorphic to $\ell^{2}$. If $\beta \in[1, \infty)$, then $D^{\beta}(M)$ is locally homeomorphic to a normed space so that $D^{\beta}(M)$ is an $A N R$.

Proof. Since $D^{\beta}(M)$ is topologically homogeneous it is enough to consider a neighborhood of the identity. Any diffeomorphism $\phi$ of $M$ that is sufficiently close to the identity can be written as $\phi(p)=\exp _{p} X_{\phi}(p)$ where exp is the $g_{1}$-exponential map and $X_{\phi}$ is a smooth vector field. The map $\phi \rightarrow X_{\phi}$ defines a topological embedding of a neighborhood of the identity of $D^{\beta}(M)$ into the linear space of smooth vector fields on $M$ with the $C^{\beta}$ topology. Conversely, every $C^{\beta}$ vector field on $M$ can be exponentiated to a $C^{\beta}$ self-map of $M$. If $M=S^{2}$, then $\phi$ fixes a triple of points if and only if $X_{\phi}$ vanishes at the points. If $M=R P^{2}$, then $\phi$ fixes a point tangent vector $v$ at $p \in M$ if and only if $X_{\phi}(p)=0$ and the differential of $X_{\phi}$ fixes $v$ (where we used that the differential of $\exp _{p}$ is the identity). We denote by $\mathcal{X}^{\beta}(M)$ the linear space of smooth vector fields on $M$ equipped with the $C^{\beta}$ norm and subject to the above vanishing conditions. Note that $\mathcal{X}^{\beta}(M)$ is normed for finite $\beta$ and Fréchet for $\beta=\infty$. Since $\beta \geq 1$ and $M$ is compact, $C^{\beta}$ diffeomorphisms form an open subset in $C^{\beta}(M, M)$, so a sufficiently small neighborhood of zero in $\mathcal{X}^{\beta}(M)$ exponentiates to a neighborhood of the identity in $D^{\beta}(M)$. If $\beta=\infty$, 
then like any separable Fréchet space $\mathcal{X}^{\beta}(M)$ is homeomorphic to $\ell^{2}$, and hence so is $D^{\infty}(M)$ being a contractible $\ell^{2}$-manifold. If $\beta$ is finite, the identity in $D^{\beta}(M)$ has a neighborhood homeomorphic to an open ball in $\mathcal{X}^{\beta}(M)$, and since any open ball and the ambient normed space are homeomorphic, the claim follows. Finally, any normed space is an AR, and any locally ANR space is an ANR, see [BP75, Section II.5].

\section{Uniformization of nonnegatively curved planes.}

Let $g_{0}$ be the standard Euclidean metric on $\mathbb{R}^{2}$, which we identify with $\mathbb{C}$. Orientation-preserving conformal automorphisms of $\mathbb{C}$ are precisely the affine maps $z \rightarrow a z+b, a, b \in \mathbb{C}$; the group acts simply transitively on pairs of distinct points of $\mathbb{C}$. Fix such a pair and let $D^{\beta}(\mathbb{C})$ be the group of smooth orientation-preserving diffeomorphisms of $\mathbb{C}$ fixing the pair and equipped with the $C^{\beta}$ topology.

As in Section 8 we conclude that $D^{\beta}(\mathbb{C})$ is a topological group, but the proof of Corollary 8.6 fails because $D^{\beta}(\mathbb{C})$ is no longer open in $C^{\beta}(\mathbb{C}, \mathbb{C})$. It is shown in $[$ Yag] that $D^{\infty}(\mathbb{C})$ is homeomorphic to $\ell^{2}$, and we adapt his argument for $\beta \in[1, \infty)$ in Lemma 11.5 to show that $D^{\beta}(\mathbb{C})$ is an ANR. Contractibility of $D^{\infty}(\mathbb{C})$ is proved in Lemma 11.2 by exhibiting a explicit deformation retraction to a point.

REMARK 9.1. The proof of contractibility of $D^{\beta}\left(S^{2}\right), \beta \notin \mathbb{Z}$, given in Section 8 does not work for $D^{\beta}(\mathbb{C})$ because metrics on $\mathbb{C}$ are not all conformally equivalent, and hence it is unclear how to define an analog of the map (8.1).

A basic property of a subharmonic function $u$ is that the limit

$$
\alpha(u):=\lim _{r \rightarrow \infty} \frac{\sup \{u(z):|z|=r\}}{\log r}
$$

exists in $[0, \infty]$. It is proved in $[\mathbf{B H 1 5}$, Theorem 1.1] that the nonnegatively curved metric $e^{-2 u} g_{0}$ is complete if and only if $\alpha(u) \leq 1$.

Let $\mathcal{O}_{\geq 0}^{\gamma}(\mathbb{C})$ be the set of smooth subharmonic functions on $\mathbb{C}$ with $\alpha(u) \leq 1$ equipped with the $C^{\gamma}$ topology, and let $\mathcal{O}_{>0}^{\gamma}(\mathbb{C})$ be the subspace of functions $u$ such that $\triangle_{g_{0}} u$ is positive. Both $\mathcal{O}_{\geq 0}^{\gamma}(\mathbb{C})$ and $\mathcal{O}_{>0}^{\gamma}(\mathbb{C})$ are convex by the proof of $[$ BH15, Lemma 2.4].

It is well known, see e.g. [BF42] that any complete nonnegative curved plane is conformally equivalent to $\left(\mathbb{C}, g_{0}\right)$. This easily implies that the map $(\phi, u) \rightarrow \phi^{*} e^{-2 u} g_{0}$ induces continuous bijections

$$
D^{\gamma+1}(\mathbb{C}) \times \mathcal{O}_{\geq 0}^{\gamma}(\mathbb{C}) \rightarrow \mathcal{R}_{\geq 0}^{\gamma}(\mathbb{C}) \quad D^{\gamma+1}(\mathbb{C}) \times \mathcal{O}_{>0}^{\gamma}(\mathbb{C}) \rightarrow \mathcal{R}_{>0}^{\gamma}(\mathbb{C})
$$

which are homeomorphisms if $\gamma \notin \mathbb{Z}$, see [BH15, Theorem 4.1] and [BH16].

Let $M$ be $S^{2}, R P^{2}$ or $\mathbb{C}$ equipped with a complete metric $g_{\kappa}$ of constant curvature $\kappa \in\{0,1\}$, and as in Section 8 we let $\Gamma^{\gamma}(M)$ denote the locally convex linear metric space of all smooth functions on $M$ with the $C^{\gamma}$ topology.

Lemma 9.3. Let $M$ be $S^{2}, R P^{2}$ or $\mathbb{C}$, and if $M=\mathbb{C}$ assume $\lambda=0$. Then $\mathcal{O}_{\geq \lambda}^{\beta}(M)$ is closed in $\Gamma^{\beta}(M)$.

Proof. The convergence of $u_{i} \in \mathcal{O}_{\geq \lambda}^{\beta}(M)$ to $u \in \Gamma^{\beta}(M)$ gives rise to the pointed 
Gromov-Hausdorff convergence $e^{-2 u_{i}} g_{\lambda} \rightarrow e^{-2 u} g_{\lambda}$ of the corresponding metrics. If $M$ is compact, then $\left(M, e^{-2 u} g_{1}\right)$ has curvature $\geq \lambda$ in the comparison sense, and hence sectional curvature $\geq \lambda$, i.e., $u \in \mathcal{O}_{\geq \lambda}^{\beta}(M)$. If $M=\mathbb{C}$, then a priori the limiting metric $e^{-2 u} g_{0}$ might not be complete, but in any case the completion of $\left(\mathbb{C}, e^{-2 u} g_{0}\right)$ is an Alexandrov space of nonnegative curvature in the comparison sense. Like any Riemannian manifold, $\left(\mathbb{C}, e^{-2 u} g_{0}\right)$ is a locally convex subset of its completion, so it is also an Alexandrov space of nonnegative curvature, and since $u$ is smooth the metric $e^{-2 u} g_{0}$ has nonnegative sectional curvature, i.e., $\triangle_{g_{0}} u \geq 0$. Since $u_{i} \in \mathcal{O}_{\geq \lambda}^{\beta}(M)$ we have $\triangle_{g_{0}} u_{i} \geq 0$ and $\alpha\left(u_{i}\right) \leq 1$. Now the proof of [BH15, Lemma 2.4] applies to show that $\alpha(u) \leq 1$ so that $u \in \mathcal{O}_{\geq 0}^{\gamma}(\mathbb{C})$.

\section{Recognizing $\ell^{2}$-manifolds: smooth topology.}

In this section we prove Theorems $1.2-1.3$ for $\gamma=\infty$. Let $M$ denote $\mathbb{C}, S^{2}$, or $R P^{2}$ equipped with a metric $g_{\kappa}$ of constant curvature $\kappa \in\{0,1\}$.

That $\mathcal{O}_{>0}^{\infty}(\mathbb{C})$ is not locally compact is proved in [BH15, Lemma 2.5] where the idea was to look at the point of $\mathbb{C}$ where the curvature of $e^{-2 u} g_{0}$ is positive and note that arbitrary small perturbations near the point rule out local compactness. The same idea works for $\mathcal{O}_{\geq \lambda}^{\infty}(M)$ when $M$ is compact. Namely, if $u$ is a constant function on $M$ such that the sectional curvature of $e^{-2 u} g_{1}$ is $>\lambda$, then the proof of [BH15, Lemma 2.5] shows that $u$ has no compact neighborhood in $\mathcal{O}_{\geq \lambda}^{\infty}(M)$.

By Lemma 9.3 the subset $\mathcal{O}_{\geq \lambda}^{\infty}(M)$ is closed in the Fréchet space $C^{\infty}(M)$. Like any closed convex non-locally-compact subset of a Fréchet space, $\mathcal{O}_{\geq \lambda}^{\infty}(M)$ is homeomorphic to $\ell^{2}$, see [DT81, Theorem 2]. Similarly, $D^{\infty}(M)$ is a contractible Fréchet manifold, hence it is homeomorphic to $\ell^{2}$, see $[\mathbf{E S 7 0}]$, [Yag], and the Fréchet space $\ell^{2} \times \ell^{2}$ is isomorphic to $\ell^{2}$. Thus $\mathcal{R}_{\geq \lambda}^{\infty}(M)$ is homeomorphic to $\ell^{2}$, where as usual $\lambda=0$ for $M=\mathbb{C}$.

If $M$ is $S^{2}$ or $R P^{2}$, then $\mathcal{O}_{>\lambda}^{\infty}(M)$ is an open contractible subset in the Fréchet space $C^{\infty}(M)$, and hence it is homeomorphic to $\ell^{2}$. It remains to prove the following.

TheOREM 10.1. $\quad \mathcal{O}_{>0}^{\infty}(\mathbb{C})$ is homeomorphic to $\ell^{2}$.

Proof. By Toruńczyk's characterization theorem, see [BRZ96, Theorem 1.1.14], a Polish AR is homeomorphic to $\ell^{2}$ if and only if it has the Strong Discrete Approximation Property (SDAP). Also a space is an AR with SDAP if and only if it is homeomorphic to a homotopy dense subset of $\ell^{2}$ [BRZ96, Theorem 1.3.2]. Note that $\mathcal{O}_{>0}^{\infty}(\mathbb{C})$ is dense in $\mathcal{O}_{>0}^{\infty}(\mathbb{C})$ : Any $u \in \mathcal{O}_{>0}^{\infty}(\mathbb{C})$ can be approximated by the convex combinations $(1-t) u+t u_{0}$ which lie in $\mathcal{O}_{>0}^{\infty}(\mathbb{C})$ for $t \in(0,1)$ provided $u_{0} \in \mathcal{O}_{>0}^{\infty}(\mathbb{C})$. If a convex subset of a linear metric space is dense in an AR, then it is homotopy dense in that AR [BRZ96, Exercises $12-13$ in 1.2]. Thus $\mathcal{O}_{>0}^{\infty}(\mathbb{C})$ is homotopy dense in $\mathcal{O}_{\geq 0}^{\infty}(\mathbb{C})$. Since $\mathcal{O}_{\geq 0}^{\infty}(\mathbb{C})$ is homeomorphic to $\ell^{2}$, it remains to check that $\mathcal{O}_{>0}^{\infty}(\mathbb{C})$ is Polish. Recall that a subspace of a Polish space is Polish if and only if it is $G_{\delta}$. To show that $\mathcal{O}_{>0}^{\infty}(\mathbb{C})$ is $G_{\delta}$ note that

$$
\mathcal{O}_{>0}^{\infty}(\mathbb{C})=\bigcap_{n}\left\{u \in \mathcal{O}_{\geq 0}^{\infty}(\mathbb{C}):\left.\triangle_{g_{0}} u\right|_{\{z:|z| \leq n\}}>0\right\}
$$


which is a countable intersection of open sets.

\section{1. $\mathcal{M}_{2}$-absorbing diffeomorphism groups.}

In this section $\beta \in[1, \infty)$ and $M$ is $S^{2}, R P^{2}$ or $\mathbb{C}$. The group $D^{\beta}(M)$ was defined in Section 8 for compact $M$ and in Section 9 for $M=\mathbb{C}$. The results of this section combine to prove the following theorem.

TheOREM 11.1. $D^{\beta}(M)$ is $\mathcal{M}_{2}$-absorbing for each $\beta \in[1, \infty)$.

Recall that any contractible $\mathcal{M}_{2}$-absorbing space is homeomorphic to $\Sigma^{\omega}$. We noted in Section 8 that $D^{\beta}\left(S^{2}\right)$ and $D^{\beta}\left(R P^{2}\right)$ are contractible for $\beta \notin \mathbb{Z}$, and $D^{\beta}\left(S^{2}\right)$ is contractible for every integer $\beta>1$.

Lemma 11.2. $D^{\beta}(\mathbb{C})$ is contractible.

Proof. Denote by $\operatorname{Diff}^{+}(\mathbb{C})$ the group of all smooth orientation-preserving diffeomorphisms of $\mathbb{C}$; we equip its subgroups with the $C^{\beta}$ topology. Let $\operatorname{Conf}^{+}(\mathbb{C})$ denote the subgroup of conformal automorphisms of $\mathbb{C}$, and let $D_{*}^{\beta}(\mathbb{C})$ be the subgroup of diffeomorphisms of $\mathbb{C}$ that fix 0 and whose differential at 0 is the identity. Again $D_{*}^{\beta}(\mathbb{C})$ is a topological group. The subgroups $D_{*}^{\beta}(\mathbb{C})$ and $D^{\beta}(\mathbb{C})$ are closed in $\operatorname{Diff}^{+}(\mathbb{C})$ and intersects $\operatorname{Conf}^{+}(\mathbb{C})$ trivially. Any element of $\operatorname{Diff}^{+}(\mathbb{C})$ can be written uniquely as the product of an element of $\mathrm{Conf}^{+}(\mathbb{C})$ with an element of either subgroup, which defines a homeomorphism of Diff ${ }^{+}(\mathbb{C})$ to the product of $\operatorname{Conf}^{+}(\mathbb{C})$ with either subgroup. The group $D_{*}^{\beta}(\mathbb{C})$ are contractible via the homotopy $H_{t}(f)(v)=f(t v) / t$ for $t \in(0,1]$ and $H_{0}(f)(v)=f_{*}(0)(v)$. Hence the inclusion $\operatorname{Conf}^{+}(\mathbb{C}) \rightarrow \operatorname{Diff}^{+}(\mathbb{C})$ is a homotopy equivalence, which implies the contractibility of $D^{\beta}(\mathbb{C})$. Indeed, the slice inclusion $D^{\beta}(\mathbb{C}) \rightarrow D^{\beta}(\mathbb{C}) \times \operatorname{Conf}^{+}(\mathbb{C})$ followed by the projection to the first factor is the identity, while the composition of the slice inclusion with the projection on the second factor, which is a homotopy equivalence, is a constant map.

Lemma 11.3. $D^{\beta}(M)$ is in $\mathcal{M}_{2}$.

Proof. Since $D^{\infty}(M)$ is homeomorphic to $\ell^{2}$, see Corollary 8.6 for compact $M$ and [Yag, Theorem 1.1] for $M=\mathbb{C}$, the space $D^{\infty}(M)$ is completely metrizable, and hence Lemma 5.2 implies the claim.

LeMma 11.4. $D^{\beta}(M)$ is $\sigma Z$.

Proof. First assume that $M$ is $S^{2}$ or $R P^{2}$. Corollary 8.6 shows that $D^{\beta}(M)$ is locally homeomorphic to the linear space $V^{\beta}$ of smooth vector fields on $M$ equipped with the $C^{\beta}$ topology. Hence $V^{\beta}$ is the image of the inclusion $\iota$ from the Fréchet space $V^{\infty}$ of smooth vector fields on $M$ to the Banach space of $C^{\beta}$ vector fields on $M$. Since $\beta$ is finite, $V^{\beta}$ is incomplete. As noted in [BDP00, Proposition 3.6] any incomplete operator image (i.e. the image of a continuous linear operator between Fréchet spaces) is $\sigma Z$. More precisely, there is an open convex neighborhood $U$ of 0 in $V^{\infty}$ such that closure $\overline{\iota(U)}$ of $\iota(U)$ in $V^{\beta}$ is a $Z$-set and so is $n \cdot \overline{\iota(U)}$ for every $n \in \mathbb{N}$. Thus $V^{\beta}$ is a 
countable union of $Z$-sets $n \cdot \overline{\iota(U)}$. Moreover, one can choose $U$ so that $\overline{\iota(U)}$ is bounded which implies that the homeomorphism of $V^{\beta}$ onto an open subset of $D^{\beta}(M)$ takes each $n \cdot \overline{\iota(U)}$ to a closed subset. Since $D^{\beta}(M)$ is separable, it is covered by countably many open sets homeomorphic to $V^{\beta}$, hence $D^{\beta}(M)$ is $\sigma Z$.

Let $M=\mathbb{C}$, and let $D$ be a closed disk embedded in $\mathbb{C}$. Fix an integer $n>\beta$, set $r=n+3$, and let $Z_{m}$ be the subspace of all $z \in D^{\beta}(\mathbb{C})$ such that for every $\rho \in(\beta, r)$ the $C^{\rho}$ norm of $\left.z\right|_{D}$ is at most $m$.

As in Lemma 12.1 below we see that $D^{\beta}(\mathbb{C})=\bigcup_{m>0} Z_{m}$, and each $Z_{m}$ is closed in $D^{\beta}(\mathbb{C})$. To show that $Z_{m}$ is a $Z$-set it remains to check that any continuous map $f$ from the Hilbert cube $Q$ into $D^{\beta}(\mathbb{C})$ is a uniform limit of maps whose ranges miss $Z_{m}$.

For $X \in\{\mathbb{C}, \overline{\mathbb{C}}\}$ let $C_{D}^{\beta}(X, X)$ be the subspace of $C^{\beta}(X, X)$ consisting of maps that are the identity outside $D$. Here $\overline{\mathbb{C}}=\mathbb{C} \cup\{\infty\}$ is the Riemann sphere. The obvious extension map $C_{D}^{\beta}(\mathbb{C}, \mathbb{C}) \rightarrow C_{D}^{\beta}(\overline{\mathbb{C}}, \overline{\mathbb{C}})$ is a homeomorphism. Given $h \in C_{D}^{\beta}(\mathbb{C}, \mathbb{C})$ we denote its extension to $\overline{\mathbb{C}}$ by $\bar{h}$. Clearly $\bar{h}$ is a $C^{\beta}$ diffeomorphism if and only if so is $h$.

For a function $p \in C^{\infty}(\mathbb{C})$ with support in $D$ we set $f_{p}(q, t)=t p+(1-t) f(q)$ where $q \in Q$ and $t \in J=[0,1)$. The associated map $f_{p}: Q \times J \rightarrow C^{\beta}(\mathbb{C}, \mathbb{C})$ is clearly continuous. We are going to show that for a suitable $p$ and small enough $t$ the map $f_{p}(\cdot, t)$ takes values in $D^{\beta}(\mathbb{C}) \backslash Z_{m}$ and approximates $f$.

The map $f_{0}(q, t)=(1-t) f(q)$ lies in $D^{\infty}(\mathbb{C})$ as the composite of $f(q)$ and the scaling by $1-t$. Set $h_{p}(q, t)=f_{0}(q, t)^{-1} \circ f_{p}(q, t)$; note that $h_{p}(q, t)$ is the identity outside $D$ for every $q, t$. Thus each $h_{p}$ gives rises to a continuous map $\bar{h}_{p}: Q \times J \rightarrow C_{D}^{\beta}(\overline{\mathbb{C}}, \overline{\mathbb{C}})$.

Fix a real-valued function $\alpha \in C^{n}(\mathbb{C})$ such that $\alpha$ is not $C^{n+1}$ and the support of $\alpha$ is in $D$. Then the map $f_{\alpha}: Q \times I \rightarrow C^{\beta}(\mathbb{C}, \mathbb{C})$ is continuous, and $f_{\alpha}(q, t)$ is not $C^{n+1}$ for each $t>0$. Note that $\bar{h}_{\alpha}(q, 0)$ is the identity for every $q$. Recall that

(i) if $L$ is a compact manifold without boundary, then $C^{1}$ diffeomorphisms of $L$ form an open subset in $C^{1}(L, L)$ [Hir94, Theorem 1.7 in Chapter 2];

(ii) a $C^{l}$ map with $l \geq 1$ is a $C^{l}$-diffeomorphism if and only if it is a $C^{1}$-diffeomorphism (by the inverse function theorem).

Since $n>\beta \geq 1$ and $Q, \overline{\mathbb{C}}$ are compact, (i)-(ii) imply that there is an $\varepsilon>0$ such that $\bar{h}_{\alpha}(\cdot, t)$ takes values in $D^{n}(\overline{\mathbb{C}})$ for all $t \in(0,2 \varepsilon)$.

Next let us approximate $\alpha$ in the $C^{n}$ topology by a sequence of functions $\alpha_{i} \in C^{\infty}(\mathbb{C})$ with supports in $D$. Since $\bar{h}_{\alpha}(q, \varepsilon) \in D^{n}(\overline{\mathbb{C}})$ and $\bar{h}_{\alpha_{i}}(q, \varepsilon)$ is $C^{\infty}$, (i)-(ii) again imply that $\bar{h}_{\alpha_{i}}(q, \varepsilon) \in D^{\infty}(\overline{\mathbb{C}})$ for all large $i$. Restricting $\bar{h}_{\alpha_{i}}(q, \varepsilon)$ to $\mathbb{C}$ and post-composing the restriction with $f_{0}(q, t)$ gives $f_{\alpha_{i}}(q, \varepsilon) \in D^{\infty}(\mathbb{C})$ for all large $i$.

By the Arzelà-Ascoli theorem the $C^{n+2}$ norm of $\left.f_{\alpha_{i}}(q, \varepsilon)\right|_{D}$ tends to infinity uniformly in $q$ (else some sequence $\left.f_{\alpha_{i}}\left(q_{i}, \varepsilon\right)\right|_{D}$ would converge to a $C^{n+1}$ map of the form $\left.f_{\alpha}(q, \varepsilon)\right|_{D}$ contradicting the choice of $\left.\alpha\right)$. In particular, the range of $f_{\alpha_{i}}(\cdot, \varepsilon)$ misses $Z_{m}$ for all large $i$. Finally, $f_{\alpha_{i}}(\cdot, \varepsilon)$ is an approximation of $f$ because $f-f_{\alpha_{i}}(\cdot, \varepsilon)=\varepsilon\left(f-\alpha_{i}\right)$ and the $C^{\beta}$ norm of $f-\alpha_{i}$ is uniformly bounded as $\alpha_{i}$ converges to $\alpha$ in the $C^{n}$ topology and $n>\beta$.

LEMma 11.5. $D^{\beta}(M)$ is homeomorphic to a homotopy dense subset of $\ell^{2}$, and in particular it is an $A N R$. 
Proof. If $M$ is $S^{2}$ or $R P^{2}$, then Corollary 8.6 implies that $D^{\beta}(M)$ is an ANR. The fact that $D^{\beta}(\mathbb{C})$ is an ANR is proved using a method of Yagasaki [Yag]. To conform to notations in $[\mathbf{Y a g}]$ denote $D^{\beta}(\mathbb{C})$ by $G$, and let $G_{K}$ denote the subgroup fixing a subset $K$ pointwise. Fix an exhaustion of $\mathbb{C}$ by closed round disks $M_{i}$ centered at 0 of radius $i>0$. Then $G_{M_{i}}$ is contractible by the Alexander trick towards infinity. Now [Yag, Theorem 3.1(2)(i)] gives a sufficient condition, called Assumption (A) in [Yag, p.8], under which the identity component of $G$ is an ANR. Since $G$ is path-connected by Lemma 11.2, it remains to check Assumption (A) which is somewhat technical to state but the two main points are as follows. The group $G_{\mathbb{C} \backslash M_{i}}$ is an ANR because it is locally homeomorphic to a normed space by the proof of Corollary 8.6. The (one paragraph) proof in $[$ Lim64] of the parametrized isotopy extension theorem extends to the setting of smooth maps with Hölder $C^{\beta}$ topology.

Finally, by [BRZ96, Theorem 4.2.1 and Theorem 1.3.2] any infinite-dimensional ANR-group is homeomorphic to a homotopy dense subset of an $\ell^{2}$-manifold, which in our case is $\ell^{2}$ because $D^{\beta}(M)$ is contractible.

LEMMA 11.6. $\quad D^{\beta}(M)$ is strongly $\mathcal{M}_{2}$-universal.

Proof. As usual we plan to establish $\mathcal{M}_{2}$-universality by applying Theorem 5.1 in which we let $N=[0,1], h_{\bullet}(x)=x, \eta=1 / 2, D=[1 / 2,2 / 3]$, and $\mathfrak{D} u=u^{\prime}$. For $n \geq 1$ let $C_{\bullet}^{n}$ be the subspace of $C^{n}([0,1])$ consisting of functions $u$ that equal $h \bullet$ outside $D$ and satisfy $u^{\prime} \geq \eta$ on $D$. By Theorem 5.1 the assumptions of Corollary 4.9 are satisfied. To apply the corollary fix $n>\beta$ and construct a map $f: C_{\bullet}^{n} \rightarrow D^{\beta}(M)$ as follows. Fix an closed coordinate disk $B$ in $M$, identify it with a unit disk in $\mathbb{C}$, and think of it in polar coordinates. Let $f(u)$ be the diffeomorphism of $M$ that is the identity outside $B$, while on $B$ it takes $r e^{i \theta}$ to $u(r) e^{i \theta}$. Clearly $f$ is injective and its continuity follows from $n>\beta$. It is easy to check that the image of $f$ is closed. Thus $D^{\beta}(M)$ is $\mathcal{M}_{2}$-universal. Clearly $D^{\beta}(M)$ is infinite dimensional, and it is an ANR by Lemma 11.5. Hence [BRZ96, Theorem 4.2.3] implies strong $\mathcal{M}_{2}$-universality of $D^{\beta}(M)$.

\section{The spaces $\mathcal{O}_{\geq \lambda}^{\gamma}(M)$ and $\mathcal{O}_{>\lambda}^{\gamma}(M)$ are $\mathcal{M}_{2}$-absorbing.}

Let $\gamma \in[0, \infty)$ and $M$ be $S^{2}, R P^{2}$ or $\mathbb{C}$ equipped with a complete metric $g_{\kappa}$ of constant curvature $\kappa \in\{0,1\}$.

In this section we adopt the following convention: whenever we write $\mathcal{O}_{\geq \lambda}^{\gamma}(\mathbb{C})$ and $\mathcal{O}_{>\lambda}^{\gamma}(\mathbb{C})$ we assume $\lambda=0$. This will allow us to treat all surfaces at once.

LEMMA 12.1. If $\gamma$ is finite, then the following spaces are $\sigma Z$

(1) $\mathcal{O}_{\geq \lambda}^{\gamma}\left(S^{2}\right), \mathcal{O}_{>\lambda}^{\gamma}\left(S^{2}\right), \mathcal{O}_{\geq \lambda}^{\gamma}\left(R P^{2}\right), \mathcal{O}_{>\lambda}^{\gamma}\left(R P^{2}\right)$ for any $\lambda \in \mathbb{R}$,

(2) $\mathcal{O}_{\geq 0}^{\gamma}(\mathbb{C})$ and $\mathcal{O}_{>0}^{\gamma}(\mathbb{C})$.

Proof. We first give a proof for $\mathcal{O}_{>\lambda}^{\gamma}(M)$ and then indicate necessary modifications for $\mathcal{O}_{\geq \lambda}^{\gamma}(M)$. Fix $r>\max \{\gamma, 2\}$, and let $D$ be a closed disk embedded in $M$. For a positive integer $m$ let $Z_{m}$ be the set of all functions $u \in \mathcal{O}_{>\lambda}^{\gamma}(M)$ such that $\left\|\left.u\right|_{D}\right\|_{C \rho(D)} \leq m$ for every $\rho \in(\max \{\gamma, 2\}, r)$. 
The equality $\mathcal{O}_{>\lambda}^{\gamma}(M)=\bigcup_{m} Z_{m}$ follows from the facts that any smooth function on $D$ has finite $C^{r}$ norm, and the Lipschitz constant of the identity map of $C^{r}(D)$, where the domain and the co-domain are given the $C^{r}$ and $C^{\rho}$ norms respectively, is bounded above independently of $\rho$, see [GT01, Lemma 6.35].

To show that $Z_{m}$ is closed consider $u_{i} \rightarrow u$ in $\mathcal{O}_{>\lambda}^{\gamma}(M)$ with $u_{i} \in Z_{m}$, and fix $\rho<\delta<r$. Since $\left\{\left.u_{i}\right|_{D}\right\}$ is uniformly bounded in the $C^{\delta}$ norm, $\left.u_{i}\right|_{D}$ subconverges in the $C^{\rho}$ topology, see [GT01, Lemma 6.36]. The limit equals $\left.u\right|_{D}$ because $\rho>\gamma$, and hence $\left\|\left.u\right|_{D}\right\|_{C^{\rho(D)}} \leq m$.

To show that $Z_{m}$ is a $Z$-set we fix a continuous map $f: Q \rightarrow \mathcal{O}_{>\lambda}^{\gamma}(M)$, where $Q$ is the Hilbert cube, and approximate it by a map whose range misses $Z_{m}$.

For the first step of the approximation consider a partition of unity $\mathfrak{u}=\left\{\psi_{i}, U_{i}\right\}$, $1 \leq i \leq k$ on $Q$ where $\psi_{i}$ is a continuous real-valued function on $Q$ with support in $U_{i}$. For each $i$ pick a point $q_{i} \in U_{i}$, and consider the function $f_{\mathfrak{u}}=\sum_{i=1}^{k} f\left(q_{i}\right) \psi_{i}$. Thus $f_{\mathfrak{u}}$ takes values in the convex hull of the functions $f\left(q_{1}\right), \ldots, f\left(q_{k}\right)$, which is a finite dimensional convex subset of $\mathcal{O}_{>\lambda}^{\gamma}(M)$. Let $\left\{p_{j}\right\}$ be a countable family of seminorms defining the topology on the ambient vector space $\Gamma^{\gamma}(M)$ with the associated metric $d=$ $\sum_{j \geq 1}\left(p_{j} /\left(p_{j}+1\right)\right) 2^{-j}$. For any $\varepsilon>0$ there is a partition of unity as above such that for any $i, j$, and $x, y \in f\left(U_{i}\right)$ we have $p_{j}(x, y)<\varepsilon$, and therefore $\sup _{q \in Q} d\left(f(q), f_{\mathfrak{u}}(q)\right)<\varepsilon$.

For the second stage of the approximation let $f_{\mathfrak{u}, \alpha}(q)=f_{\mathfrak{u}}(q)+\alpha$ where $\alpha$ is a real valued smooth function on $M$ supported in $D$ and such that $\left\|\left.\alpha\right|_{D}\right\|_{C^{\gamma}(D)}$ and $\left\|\left.\alpha\right|_{D}\right\|_{C^{2}(D)}$ are small. If $M=\mathbb{C}$, then for each $q$ the functions $f_{\mathfrak{u}, \alpha}(q)$ and $f_{\mathfrak{u}}(q)$ have the same growth at infinity as $\alpha$ is compactly supported. Compactness of $Q$ and strictness of the inequality " $>\lambda$ " makes the image of $f_{\mathfrak{u}, \alpha}$ lie in $\mathcal{O}_{>\lambda}^{\gamma}(M)$ provided $\left\|\left.\alpha\right|_{D}\right\|_{C^{2}(D)}$ is sufficiently small.

The map $\left.q \rightarrow f_{\mathfrak{u}}(q)\right|_{D}$ takes values in a finite dimensional vector subspace of $\Gamma^{\gamma}(D)$, and is continuous with respect to the $C^{\gamma}$ norm on the co-domain, and hence with respect to any norm, as they are all equivalent. In particular, compactness of $Q$ implies that there is $R>0$ with $\left\|\left.f_{\mathfrak{u}}(q)\right|_{D}\right\|_{C^{\rho}(D)} \leq R$ for each $q \in Q$ and every $\rho \in(\max \{\gamma, 2\}, r)$.

We can also choose $\alpha$ so that $\left\|\left.\alpha\right|_{D}\right\|_{C^{\sigma}(D)}>m+R$ for some $\sigma \in(\max \{\gamma, 2\}, r)$. Then the image of $f_{\mathfrak{u}, \alpha}$ is disjoint from $Z_{m}$ for if $f_{\mathfrak{u}, \alpha}(q) \in Z_{m}$ for some $q$, then

$$
\left\|\left.\alpha\right|_{D}\right\|_{C^{\sigma}(D)}=\left\|\left.f_{\mathfrak{u}, \alpha}(q)\right|_{D}-\left.f_{\mathfrak{u}}(q)\right|_{D}\right\|_{C^{\sigma}(D)} \leq m+R .
$$

To treat the case of $\mathcal{O}_{\geq \lambda}^{\gamma}(M)$ we need one more step in the approximation. Fix any function $v \in \mathcal{O}_{>\lambda}^{\gamma}(M)$. (If $M$ is compact, we can let $v$ be a sufficiently large constant such that $e^{2 v}>\lambda$, and if $M=\mathbb{C}$, then take $v$ be any function such that $e^{-2 v} g_{0}$ has a complete metric of positive curvature). Now any continuous map $f: Q \rightarrow \mathcal{O}_{\geq \lambda}^{\gamma}(M)$ can be approximated by the map $q \rightarrow(1-t) f(q)+t v$ for small positive $t$, and the image of the latter map lies in $\mathcal{O}_{>\lambda}^{\gamma}(M)$ (see the proof of Lemma 8.2 and note that the leftmost inequality in the displayed formula applied to $(1-t) f(q)+t v$ is strict for $t \in(0,1)$ because $\left.v \in \mathcal{O}_{>\lambda}^{\gamma}(M)\right)$.

LEMMA 12.2. Each space in the statement of Lemma 12.1 belongs to $\mathcal{M}_{2}$.

Proof. By Lemma 5.2 it suffices to show that the corresponding spaces with $\gamma=\infty$ are completely metrizable. A subspace of a complete metric space is completely 
metrizable if and only if it is $G_{\delta}$. The latter is true for $\mathcal{O}_{>0}^{\infty}(\mathbb{C})$ because it is $G_{\delta}$ in the ambient Fréchet spaces by the proof of Theorem 10.1. If $M$ is $S^{2}$ or $R P^{2}$, then $\mathcal{O}_{\geq \lambda}^{\infty}(M)$ and $\mathcal{O}_{>\lambda}^{\infty}(M)$ are respectively closed and open, and hence are $G_{\delta}$. Finally, $\mathcal{O}_{\geq 0}^{\infty}(\mathbb{C})$ is closed by [BH15, Lemma 2.4].

Lemma 12.3. Each space in the statement of Lemma 12.1 is $\mathcal{M}_{2}$-universal.

Proof. Recall that a space is $\mathcal{M}_{2}$-universal if it contains an $\mathcal{M}_{2}$-universal closed subset, and to find one we are going to apply Corollary 4.9 and Theorem 5.1. To satisfy the assumptions of Theorem 5.1 let $N=M, l=2, \mathfrak{D} u=e^{2 u}\left(\kappa+\triangle_{g_{\kappa}}\right)$, fix a function $h_{\bullet} \in \mathcal{O}_{>\lambda}^{\gamma}(M)$, fix a closed coordinate disk $D$ in $M$, and let $\eta$ be a constant such that $\max \{0, \lambda\}<\eta<\left.\min \mathfrak{D} h_{\bullet}\right|_{D}$. With these data for $n \geq \max \{l, \gamma\}$ define $C_{\bullet}^{n}$ as in Theorem 5.1, i.e., let $C_{\bullet}^{n}$ denote the subspace of $C^{n}(M)$ of functions $u$ such that $\left.\mathfrak{D} u\right|_{D} \geq \eta$ and $\left.u\right|_{M \backslash \text { Int } D}=h_{\bullet}$. Corollary 4.9 applied to the inclusion of $C_{\bullet}^{n}$ into $\Gamma^{\gamma}(M)$ shows that $C_{\bullet}^{\infty}=\bigcap_{n} C_{\bullet}^{n}$ is $\mathcal{M}_{2}$-universal. Using the proof of Lemma 9.3 we see that that $C_{\bullet}^{\infty}=\bigcap_{n} C_{\bullet}^{n}$ is closed in $\Gamma^{\gamma}(M)$, and by construction $C_{\bullet}^{\infty}$ lies on $\mathcal{O}_{>\lambda}^{\gamma}(M)$.

TheOREM 12.4. Each space in the statement of Lemma 12.1 is $\mathcal{M}_{2}$-absorbing, and in particular, is homeomorphic to $\Sigma^{\omega}$.

Proof. Let $\bar{\Gamma}^{\gamma}(M)$ be the closure of $\Gamma^{\gamma}(M)$ in the Fréchet space of all $C^{\gamma}$ functions on $M$ with the $C^{\gamma}$ topology; thus $\bar{\Gamma}^{\gamma}(M)$ is a separable Fréchet space. The closure of $\mathcal{O}_{>\lambda}^{\gamma}(M)$ in $\bar{\Gamma}^{\gamma}(M)$ is not contained in Aff $\mathcal{O}_{>\lambda}^{\gamma}(M)$ because it clearly contains some nonsmooth rotationally symmetric functions; the same therefore holds for $\mathcal{O}_{\geq \lambda}^{\gamma}(M)$. Hence by Lemma $3.1 \mathcal{O}_{>\lambda}^{\gamma}(M), \mathcal{O}_{\geq \lambda}^{\gamma}(M)$ are homeomorphic to homotopy dense subsets of $\ell^{2}$. The proof of Lemma 12.3 actually shows that the $\mathcal{M}_{2}$-universal subset $f\left(C_{\bullet}^{\infty}\right)$ is closed in $\Gamma^{\gamma}(M)$. Therefore [BRZ96, Proposition 5.3.5] implies that $\mathcal{O}_{>\lambda}^{\gamma}(M), \mathcal{O}_{>\lambda}^{\gamma}(M)$ are strongly $\mathcal{M}_{2}$-universal. Thus Lemmas $12.1,12.2$, imply that $\mathcal{O}_{>\lambda}^{\gamma}(M), \mathcal{O}_{\geq \lambda}^{\gamma}(M)$ are $\mathcal{M}_{2}$-absorbing.

\section{References}

[AC92] M. T. Anderson and J. Cheeger, $C^{\alpha}$-compactness for manifolds with Ricci curvature and injectivity radius bounded below, J. Differential Geom., 35 (1992), 265-281.

[Ban00] T. Banakh, Operator images homeomorphic to $\Sigma^{\omega}$, Dissertationes Math. (Rozprawy Mat.), 387 (2000), 53-81.

[BC00] T. Banakh and R. Cauty, Interplay between strongly universal spaces and pairs, Dissertationes Math. (Rozprawy Mat.), 386 (2000), 38.

[BDP00] T. Banakh, T. Dobrowolski and A. Plichko, The topological and Borel classification of operator images, Dissertationes Math. (Rozprawy Mat.), 387 (2000), 37-52.

[BF42] C. Blanc and F. Fiala, Le type d'une surface et sa courbure totale, Comment. Math. Helv., 14 (1941-42), 230-233.

[BH15] I. Belegradek and J. Hu, Connectedness properties of the space of complete nonnegatively curved planes, Math. Ann., 362 (2015), 1273-1286.

[BH16] I. Belegradek and J. Hu, Erratum to: Connectedness properties of the space of complete nonnegatively curved planes, Math. Ann., 364 (2016), 711-712.

[BHS05] B. Bojarski, P. Hajlasz and P. Strzelecki, Sard's theorem for mappings in Hölder and Sobolev spaces, Manuscripta Math., 118 (2005), 383-397. 
[BM86] M. Bestvina and J. Mogilski, Characterizing certain incomplete infinite-dimensional absolute retracts, Michigan Math. J., 33 (1986), 291-313.

[BP75] C. Bessaga and A. Pelczyński, Selected topics in infinite-dimensional topology, Monografie Matematyczne, Tom 58, [Mathematical Monographs, 58], PWN-Polish Scientific Publishers, Warsaw, 1975.

[BRZ96] T. Banakh, T. Radul and M. Zarichnyi, Absorbing sets in infinite-dimensional manifolds, Mathematical Studies Monograph Series, 1, VNTL Publishers, L'viv, 1996.

[CDM93] R. Cauty, T. Dobrowolski and W. Marciszewski, A contribution to the topological classification of the spaces $C_{p}(X)$, Fund. Math., 142 (1993), 269-301.

[DM90] T. Dobrowolski and J. Mogilski, Problems on topological classification of incomplete metric spaces, Open problems in topology, North-Holland, Amsterdam, 1990, 409-429.

[Don11] S. Donaldson, Riemann surfaces, Oxford Graduate Texts in Mathematics, 22, Oxford University Press, Oxford, 2011.

[DT81] T. Dobrowolski and H. Toruńczyk, Separable complete ANRs admitting a group structure are Hilbert manifolds, Topology Appl., 12 (1981), 229-235.

[ES70] C. J. Earle and A. Schatz, Teichmüller theory for surfaces with boundary, J. Differential Geometry, 4 (1970), 169-185.

[FM78] H. D. Fegan and R. S. Millman, Quadrants of Riemannian metrics, Michigan Math. J., 25 (1978), 3-7.

[GT01] D. Gilbarg and N. S. Trudinger, Elliptic partial differential equations of second order, Classics in Mathematics, Springer-Verlag, Berlin, 2001, reprint of the 1998 edition.

[Hir94] M. W. Hirsch, Differential topology, Graduate Texts in Mathematics, 33, Springer-Verlag, New York, 1994, Corrected reprint of the 1976 original.

[Hu15] J. Hu, Complete nonnegatively curved spheres and planes, Ph.D. thesis, Georgia Tech, 2015.

[Kur66] K. Kuratowski, Topology, I, New edition, revised and augmented, Translated from the French by J. Jaworowski, Academic Press, New York-London; Państwowe Wydawnictwo Naukowe, Warsaw, 1966.

[KW74] J. L. Kazdan and F. W. Warner, Curvature functions for compact 2-manifolds, Ann. of Math. (2), 99 (1974), 14-47.

[Lim64] E. L. Lima, On the local triviality of the restriction map for embeddings, Comment. Math. Helv., 38 (1964), 163-164.

[Mog84] J. Mogilski, Characterizing the topology of infinite-dimensional $\sigma$-compact manifolds, Proc. Amer. Math. Soc., 92 (1984), 111-118.

[Pal68] R. S. Palais, Foundations of global non-linear analysis, W. A. Benjamin, Inc., New YorkAmsterdam, 1968.

[Pet97] P. Petersen, Convergence theorems in Riemannian geometry, Comparison geometry (Berkeley, CA, 1993-94), Math. Sci. Res. Inst. Publ., 30, Cambridge Univ. Press, Cambridge, 1997, 167-202.

[Sma59] S. Smale, Diffeomorphisms of the 2-sphere, Proc. Amer. Math. Soc., 10 (1959), 621-626.

[Trè67] F. Trèves, Topological vector spaces, distributions and kernels, Academic Press, New York, 1967.

[Yag] T. Yagasaki, Homotopy types of diffeomorphism groups of noncompact 2-manifolds, arXiv:math/0109183v3.

\section{Taras BANAKH}

Ivan Franko National University of Lviv Ukraine

and Jan Kochanowski University in Kielce

Poland

E-mail: t.o.banakh@gmail.com

\section{Igor BELEGRADEK}

School of Mathematics

Georgia Institute of Technology

Atlanta

GA 30332-0160, USA

E-mail: ib@math.gatech.edu 